\title{
Conditions for Well-Posedness in Gevrey Classes of the Cauchy Problems for Fuchsian Hyperbolic Operators
}

By

\author{
Hitoshi URYU*
}

\section{§1. Introduction}

Let us consider the following operator.

$$
P=\partial_{t}^{2}-t^{2 l} \partial_{x}^{2}+a t^{s} \partial_{x}
$$

where $l$ and $s$ are non-negative integers and $a$ is non-zero constant. It is wellknown that if $s \geqq l-1$, the Cauchy problem for $P$ is well-posed in $C^{\infty}$ (see Oleinik [11]). Ivrii [6] showed the following. When $0 \leqq s<l-1$, the Cauchy problem for $P$ is well-posed in Gevrey class $\gamma_{l o c}^{(\kappa)}$ if and only if $1 \leqq \kappa<(2 l-s) /$ $(l-s-1)$. This simple example shows us a delicate relation among the wellposed class, the order of degeneracy of a principal part and that of a lower order term for non-strictly hyperbolic operators. Hence in this paper we shall consider whether this fact is valid for more general non-strictly hyperbolic operators.

In the case of non-characteristic operators the well-posedness in Gevrey class is studied by Ohya [10], Leray and Ohya [9], Beals [1], Bronstein [3], Ivrii [5], Kajitani [7], Komatsu [8], Steinberg [12], Trepreau [14], Wakabayashi [17] and others. Igari [4] extends Ivrii's example to higher order non-strictly hyperbolic operators with double characteristics under some assumptions on coefficients of the operators.

On the other hand Baouendi and Goulaouic [2] define Fuchsian partial differential operators and discuss Cauchy Kowalevski's type theorem. Tahara

Communicated by S. Matsuura, January 18, 1983.

* Department of Mathematics, School of Science and Engineering, Waseda University, Tokyo 160, Japan.

Present address: School of Business Administration, Senshu University, Kanagawa 214, Japan. 
[13] considers the Cauchy problems for Fuchsian hyperbolic operators in smooth function space. Here we shall note that Fuchsian partial differential operators are the natural extension of non-characteristic operators.

Hence we shall discuss the well-posedness of the Cauchy problems for Fuchsian hyperbolic operators in the Gevrey class. And we shall get a close connection among an admissible class of the Cauchy problem, a principal part and lower order terms.

Let $\quad(t, x) \in[0, T] \times \mathbb{R}^{n} \quad$ and $\quad\left(D_{t}, D_{x}\right)=\left(D_{t}, D_{x_{1}}, \ldots, D_{x_{n}}\right)=\left(-\sqrt{-1} \partial_{t}\right.$, $\left.-\sqrt{-1} \partial_{x_{1}}, \ldots,-\sqrt{-1} \partial_{x_{n}}\right)$. Let us denote by $(\tau, \xi)$ the dual variable of $(t, x)$. Next we shall define function spaces used in this paper.

Definition 1. $\left(\gamma_{\text {loc }}^{(\kappa)} ; \kappa \geqq 1\right)$ We define $\gamma_{l o c}^{(\kappa)}$ the set of functions $f(x) \in$ $C^{\infty}\left(\boldsymbol{R}^{n}\right)$ satisfying the property that for any compact set $K \subset \boldsymbol{R}^{n}$ there exist constants $c, R>0$ such that for any multi-indices $\alpha$

$$
\left|D_{x}^{\alpha} f(x)\right| \leqq c R^{|\alpha|}(|\alpha| !)^{\kappa} \quad \text { for } \quad x \in K .
$$

Definition 2. $\left(\gamma^{(\kappa)} ; \kappa \geqq 1\right)$ We denote by $\gamma^{(\kappa)}$ the set of functions $f(x) \in$ $C^{\infty}\left(\boldsymbol{R}^{n}\right)$ with the following property. There exist constants $c, R>0$ such that for any multi-indices $\alpha$

$$
\left|D_{x}^{\alpha} f(x)\right| \leqq c R^{|\alpha|}(|\alpha| !)^{\kappa} \quad \text { for } \quad x \in \mathbb{R}^{n} .
$$

Definition 3. $\left(\Gamma^{(\kappa)} ; \kappa \geqq 1\right) \quad$ We say $f(x) \in H^{\alpha} \quad\left(\equiv \bigcap_{s} H^{s}\left(\boldsymbol{R}^{n}\right)\right)$ belongs to $\Gamma^{(\kappa)}$ if there exist constants $c, R>0$ such that

$$
\left\|D_{x}^{\alpha} f(x)\right\| \leqq c R^{|\alpha|}(|\alpha| !)^{\kappa}
$$

for any multi-indices $\alpha \in N^{n}$, where \|\| is $L^{2}$-norm with respect to $x$.

Now we shall define Fuchsian partial differential operators according to Baouendi-Goulaouic [2]. Let

$$
\begin{aligned}
P\left(t, x, D_{t}, D_{x}\right)= & t^{h} D_{t}^{m}+P_{1}\left(t, x, D_{x}\right) t^{h-1} D_{t}^{m-1}+\cdots \\
& +P_{k}\left(t, x, D_{x}\right) D_{t}^{m-k}+P_{k+1}\left(t, x, D_{x}\right) D_{t}^{m-k-1}+\cdots+P_{m}\left(t, x, D_{x}\right)
\end{aligned}
$$

be a partial differential operator satisfying the following.
$(\mathrm{A}-\mathrm{I})$
$k \in \mathbb{Z}, \quad 0 \leqq k \leqq m$

$$
\text { ord } P_{j}\left(t, x, D_{x}\right) \leqq j
$$$$
\text { ord } P_{j}\left(0, x, D_{x}\right)=0 \quad \text { for } \quad 1 \leqq j \leqq k
$$

Then $P$ is said to be of Fuchsian type with weight $m-k$ with respect to $t$. From (A-III) we shall set $P_{j}\left(0, x, D_{x}\right)=a_{j}(x)$ for $1 \leqq j \leqq k$. Let $\mathscr{C}(\lambda, x)$ be a charac- 
teristic polynomial

$$
\begin{aligned}
\mathscr{C}(\lambda, x)=\lambda(\lambda-1) \cdots(\lambda-m+1)+ & i a_{1}(x) \lambda(\lambda-1) \cdots(\lambda-m+2) \\
& +\cdots+i^{k} a_{k}(x) \lambda(\lambda-1) \cdots(\lambda-m+k+1) .
\end{aligned}
$$

Its roots, called characteristic exponents, are denoted by $\lambda=0,1, \ldots, m-k-1$, $\rho_{1}(x), \ldots, \rho_{k}(x)$.

(A-IV) there exists a constant $c>0$ such that

$$
\left|\left(\lambda-\rho_{1}(x)\right) \cdots\left(\lambda-\rho_{k}(x)\right)\right| \geqq c / \lambda(\lambda-1) \cdots(\lambda-m+k+1)
$$

for $\lambda \in \mathbb{Z}, \lambda \geqq m-k$.

Under these assumptions, we can consider the following Cauchy problem for $P$

$$
\left\{\begin{array}{l}
P\left(t, x, D_{t}, D_{x}\right) u(t, x)=f(t, x) \\
\left.D_{t}^{j} u(t, x)\right|_{t=0}=u_{j}(x), \quad 0 \leqq j \leqq m-k-1 .
\end{array}\right.
$$

Baouendi-Goulaouic [2] study the Cauchy problem for $P$ in the analytic function space and Tahara [13] investigate in $C^{\infty}$-function space. Since our function space is Gevrey class, we assume that coefficients of $P$ belong to $\mathscr{B}\left([0, T], \gamma^{(\kappa)}\right)$ i.e.

$$
P_{j}\left(t, x, D_{x}\right)=\sum_{|\beta| \leqq j} a_{j, \beta}(t, x) D_{x}^{\beta}
$$

where $a_{j, \beta}(t, x) \in \mathscr{B}\left([0, T], \gamma^{(\kappa)}\right)$.

Next we shall consider a leading term of $P$.

$$
\begin{aligned}
& \tau^{m}+\sum_{j=1}^{k} \sum_{|\beta|=j} a_{j, \beta}(t, x) \tau^{m-j} \xi^{\beta}+\sum_{j=k^{+}}^{m} \sum_{|\beta|=j} a_{j, \beta}(t, x) \\
& \times t^{j-k} \tau^{m-j} \xi^{\beta}=\prod_{j=1}^{m}\left(\tau-t^{l} \lambda_{j}(t, x, \xi)\right)
\end{aligned}
$$

where $l>0$ is a rational number and $\lambda_{j}(t, x, \check{\zeta})$ are real valued functions with the property:

If $i \neq j, \lambda_{i} \neq \lambda_{j}$ for any $(t, x) \in[0, T] \times \mathbb{R}^{n},|\xi|=1$ and for any $b \geqq 0, \alpha, \beta \in \mathbb{N}^{n}$ there exists a constant $c=c_{\alpha, \beta, b}$ such that

$$
\left|D_{\xi}^{\alpha} D_{t}^{b} D_{x}^{\beta} \lambda_{j}(t, x, \xi)\right| \leqq c \quad \text { for }(t, x) \in[0, T] \times \mathbb{R}^{n},|\xi|=1 .
$$

Finally we shall assume on lower order terms of $P$.

(A-VII) For $1 \leqq|\beta| \leqq j-1,2 \leqq j \leqq m$

$$
a_{j, \beta}(t, x)=t^{\gamma(j, \beta)} \hat{a}_{j, \beta}(t, x)
$$

where $\gamma(j, \beta)$ is a non-negative integer and $\hat{a}_{j, \beta}(t, x) \in \mathscr{B}\left([0, T], \gamma^{(\kappa)}\right)$. 
We can easily see from (A-III) that $\gamma(j, \beta) \geqq 1$ for $2 \leqq j \leqq k, 1 \leqq|\beta| \leqq j-1$. Here we shall define a number as follows.

$$
\alpha(m-j+|\beta|, \beta)= \begin{cases}\gamma(j, \beta) & \text { if } \quad 2 \leqq j \leqq k \\ \gamma(j, \beta)+j-k & \text { if } \quad k+1 \leqq j \leqq m .\end{cases}
$$

Let us note that $\alpha(m-j+|\beta|, \beta) \geqq 1$.

Here we shall define the important number $\sigma \geqq 1$ which determines admissible data classes of the Cauchy problems. For any $j(1 \leqq j \leqq m-1)$ let $k_{j}\left(1 \leqq k_{j}\right.$ $\leqq m-1)$ be the lowest integer such that $\alpha(j, \beta) / l-|\beta|+k_{j}>0$ for any $\beta(1 \leqq|\beta|$ $\leqq j-1)$. Next we set

$$
\sigma_{j}=\max _{1 \leqq|\beta| \leqq j-1}\{|\beta|-\alpha(j, \beta) / l, 0\} \quad \text { and } \quad v=\max _{1 \leqq i \leqq m-1}\left\{\sigma_{i} / k_{i}\right\} .
$$

Next we define $\sigma \geqq 1$ such that

$$
\sigma=\max _{1 \leqq i \leqq m-1}\left\{\left(k_{i} v+m-i\right) /(m-i)\right\} .
$$

Then we obtain the main theorem.

Theorem 1. Under the assumptions (A-I) (A-VII), for any $u_{j}(x) \in \gamma_{\text {loc }}^{(\kappa)}$ $(0 \leqq j \leqq m-k-1)$ and for any $f(t, x) \in \mathscr{B}\left([0, T], \gamma_{\text {loc }}^{(\kappa)}\right)$ there exists an unique solution $u(t, x) \in \mathscr{B}\left([0, T], \gamma_{\text {loc }}^{(\kappa)}\right)$ of the equation

$$
\left\{\begin{array}{l}
P\left(t, x, D_{t}, D_{x}\right) u(t, x)=f(t, x) \\
\left.D_{t}^{j} u(t, x)\right|_{t=0}=u_{j}(x), \quad 0 \leqq j \leqq m-k-1
\end{array}\right.
$$

for any $\kappa(1 \leqq \kappa<\sigma /(\sigma-1))$, i.e. the Cauchy problem (1.7) is well-posed in $\gamma_{\text {loc }}^{(\kappa)}$ $(1 \leqq \kappa<\sigma /(\sigma-1))$.

Note. (1) In the case of $\sigma=1$ the Cauchy problem (1.7) is well-posed in $C^{\infty}$-function space (see Tahara [13]). (2) From the definition of $v$ we have $0 \leqq v<1$.

Finally we shall state some examples of Theorem 1.

Example 1. Let $P$ be a second order partial differential operator

$$
P=D_{t}^{2}-t^{2 l} D_{x}^{2}+a(t, x) D_{t}+b(t, x) t^{s} D_{x}+c(t, x)
$$

where $l, s$ are non-negative integers and coefficients $a, b, c$ belong to $\mathscr{B}([0, T]$, $\left.\gamma^{(\kappa)}\right)$. In the case of $s \geqq l-1$ the Cauchy problem for $P$ is well-posed in $\gamma_{l o c}^{(\kappa)}$ $(1 \leqq k)$. When $0 \leqq s<l-1$ the Cauchy problem for $P$ is well-posed in $\gamma_{l o c}^{(\kappa)}$ $(1 \leqq \kappa<(2 l-s) /(l-s-1))$. 
Example 2. Let $P$ be a Fuchsian hyperbolic operator satisfying (A-IV)

$$
P=t^{2} D_{t}^{2}-t^{2 l} D_{x}^{2}+a(t, x) t D_{t}+b(t, x) t^{s} D_{x}+c(t, x)
$$

where $l, s$ are positive integers and $a, b, c \in \mathscr{B}\left([0, T], \gamma^{(\kappa)}\right)$. If $s \geqq l$ the Cauchy problem for $P$ is well-posed in $\gamma_{l o c}^{(\kappa)}$ for any $\kappa \geqq 1$. In the case of $0 \leqq s<l, \gamma_{l o c}^{(\kappa)}$ $(1 \leqq \kappa<(2 l-s) /(l-s))$ is admissible data classes of the Cauchy problem for $P$. Next example is the generalization of example 1.

Example 3. Let $P=P\left(t, x, D_{t}, D_{x}\right)$ be an operator of orer $m$ whose coefficients belong to $\mathscr{B}\left([0, T], \gamma^{(\kappa)}\right)$,

$$
P=P_{m}+P_{m-1}+\cdots+P_{0} .
$$

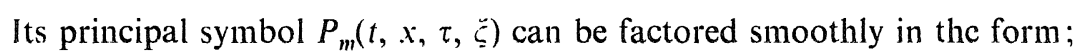

$$
P_{m}(t, x, \tau, \varsigma)=\prod_{j=1}^{m}\left(\tau-t^{\prime} \lambda_{j}(t, x, \xi)\right)
$$

where $l$ is non-negative integer, $\lambda_{j}$ is real valued function and $\lambda_{i} \neq \lambda_{j}$ when $i \neq j$. Furthermore for any $b \geqq 0$ and any multi-indices $\alpha, \beta$ there exists a constant $c=c_{\alpha, \beta, b}$ such that

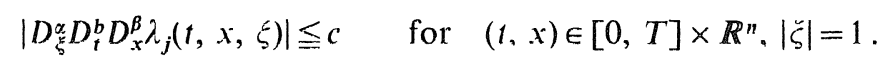

We assume that each lower order term $P_{i}(1, x, \tau, \zeta)(0 \leqq i \leqq m-1)$ is represented as follows.

$$
P_{i}(t, x, \tau, \breve{\zeta})=\sum_{|\beta|=0}^{i} a_{i, \beta}(t, x) t^{s(i, \beta)} \tau^{i-|\beta| \xi^{\beta}}
$$

where $s(i, \beta)$ is a non-negative integer and $a_{i, \beta}(t, x) \in \mathscr{B}\left([0, T], \gamma^{(h)}\right)$. Then we have easily seen that $\alpha(i, \beta)=s(i, \beta)+m-i+|\beta|$. Therefore applying Theorem 1 , we can obtain admissible data classes of the Cauchy problem for $P$.

\section{§. Sketch of the Proof of Theorem $\mathbb{1}$}

Let us start with the following theorem.

Theorem 2. Under the assumptions $(\mathrm{A}-\mathrm{I}) \sim(\mathrm{A}-\mathrm{VII})$, assertions $\mathrm{I}$ and 2 are realized.

$1^{\circ}$ For any $u_{i}(x) \in \Gamma^{(\kappa)}(0 \leqq i \leqq m-k-1)$ and any $f(t, x) \in \mathscr{B}\left([0, T], \Gamma^{\left({ }^{k}\right)}\right)$ there exists a unique solution $u(t, x) \in \mathscr{B}\left([0, T], \Gamma^{(\kappa)}\right)$ of $(1.7)$ for any $1 \leqq k$ $<\sigma /(\sigma-1)$. 
$2^{\circ} \quad$ If $\operatorname{supp}\left(u_{i}(x)\right) \subset K(0 \leqq i \leqq m-k-1)$ and $\operatorname{supp}(f(t, x)) \subset C_{l}(K)$ hold for some compact set $K \subset \boldsymbol{R}^{n}, u(t, x)$ also satisfies supp $(u(t, x)) \subset C_{l}(K)$.

Here we denote by $C_{l}(K)$

where

$$
C_{l}(K)=\left\{(t, x) \in[0, T] \times \boldsymbol{R}^{n}, \min _{y \in K}|x-y| \leqq \lambda_{\max }|t|^{l} / l\right\}
$$

$$
\lambda_{\max }=\max \left\{\left|\lambda_{i}(t, x, \xi)\right| ;(t, x, \xi) \in[0, T] \times \boldsymbol{R}^{n} \times \boldsymbol{R}^{n},|\xi|=1\right\} .
$$

Now we shall show that Theorem 2 implies Theorem 1 .

Proof of Theorem 1. We shall begin with the existence of a solution of (1.7). Let $\left\{\phi_{p}(x)\right\}$ be a partition of unity. Namely, $\phi_{p}(x)$ is compactly supported $\gamma^{(\kappa)}$ functions satisfying (i) $0 \leqq \phi_{p}(x) \leqq 1$, (ii) the summation $\sum \phi_{p}(x)$ is locally finite and (iii) $\sum \phi_{p}(x)=1$ on $\boldsymbol{R}^{n}$. For any $u_{i}(x) \in \gamma_{\text {loc }}^{(\kappa)}(0 \leqq i \leqq m-k-1)$ and any $f(t, x) \in \mathscr{B}\left([0, T], \gamma_{\text {loc }}^{(\kappa)}\right)$ we set $u_{p}^{i}(x)=\phi_{p}(x) u_{i}(x), f_{p}(t, x)=\phi_{p}(x) \times$ $f(t, x)$. Then we can easily see $u_{p}^{i}(x) \in \Gamma^{(\kappa)}$ and $f_{p}(t, x) \in \mathscr{B}\left([0, T], \Gamma^{(\kappa)}\right)$. Therefore from $1^{\circ}$ of Theorem 2 we can find a solution $u_{p}(t, x) \in \mathscr{B}\left([0, T], \Gamma^{(\kappa)}\right)$ of the equation

$$
\left\{\begin{array}{l}
P\left(t, x, D_{t}, D_{x}\right) u_{p}(t, x)=f_{p}(t, x) \\
\left.D_{t}^{i} u_{p}(t, x)\right|_{t=0}=u_{p}^{i}(x), \quad 0 \leqq i \leqq m-k-1 .
\end{array}\right.
$$

From Sobolev's lemma we have $\Gamma^{(\kappa)} \subset \gamma^{(\kappa)}$. Therefore solutions $u_{p}(t, x) \in$ $\mathscr{B}\left([0, T], \gamma^{(\kappa)}\right)$. Furthermore since the summation $\sum u_{p}(t, x)$ is locally finite, the function $u(t, x)=\sum u_{p}(t, x)$ belongs to $\mathscr{B}\left([0, T], \gamma_{\text {loc }}^{(\kappa)}\right)$ and satisfies the equation (1.7).

Secondly we shall consider the uniqueness of the solutions. Let $u(t, x)$ $\in \mathscr{B}\left([0, T], \gamma_{l o c}^{(\kappa)}\right)$ be a solution of the equation

$$
\left\{\begin{array}{l}
P\left(t, x, D_{t}, D_{x}\right) u(t, x)=0 \\
\left.D_{t}^{i} u(t, x)\right|_{t=0}=0, \quad 0 \leqq i \leqq m-k-1 .
\end{array}\right.
$$

Following Tahara [13], we shall show $u(t, x) \equiv 0$ for $(t, x) \in[0, T] \times \boldsymbol{R}^{n}$ by two steps. The first step is to prove that $u(t, x)=0$ in a neighbourhood of $\{0\} \times \boldsymbol{R}^{n}$. Let $\phi(x)$ be a compactly supported $\gamma_{l o c}^{(\kappa)}$-function such that $\phi(x)=1$ in a neighbourhood of some point $x_{0} \in \mathbb{R}^{n}$. Then $P\left(t, x, D_{t}, D_{x}\right) \phi(x) u(t, x) \in \mathscr{B}([0, T]$, $\left.\Gamma^{(\kappa)}\right)$. Therefore the solution $w(t, x)$ of

$$
\left\{\begin{array}{l}
P\left(t, x, D_{t}, D_{x}\right) w(t, x)=P\left(t, x, D_{t}, D_{x}\right) \phi(x) u(t, x) \\
\left.D_{t}^{i} w(t, x)\right|_{t=0}=0, \quad 0 \leqq i \leqq m-k-1
\end{array}\right.
$$


can be found in $\mathscr{B}\left([0, T], \Gamma^{(\kappa)}\right)$ and from $2^{\circ}$ of Theorem $2 w(t, x)=0$ in a neighbourhood of $\left(0, x_{0}\right)$. Here put $\tilde{u}(t, x)=\phi(x) u(t, x)-w(t, x)$. Then from the above the function $\tilde{u}(t, x)$ satisfies the equation

$$
\left\{\begin{array}{l}
P\left(t, x, D_{t}, D_{x}\right) \tilde{u}(t, x)=0 \\
\left.D_{t}^{i} \tilde{u}(t, x)\right|_{t=0}=0, \quad 0 \leqq i \leqq m-k-1
\end{array}\right.
$$

and $\tilde{u}(t, x)=u(t, x)$ in a neighbourhood of $\left(0, x_{0}\right)$. Hence it follows from $1^{\circ}$ of Theorem 2 that $\tilde{u}(t, x)=0$ in $[0, T] \times \mathbb{R}^{n}$. Therefore $u(t, x)=0$ in a neighbourhood of $\left(0, x_{0}\right)$. The second step is to show the uniqueness in $[0, T] \times \mathbb{R}^{n}$. Take any $\left(t_{0}, x_{0}\right) \in(0, T] \times \mathbb{R}^{n}$ and put $K=\overline{D_{l}\left(t_{0}, x_{0}\right)} \cap\{t=0\}$ where $D_{l}\left(t_{0}, x_{0}\right)$ $=\left\{(t, x) \in[0, T] \times \mathbb{R}^{n} ;\left|x-x_{0}\right|<\lambda_{\max }\left(t_{0}^{l}-t^{l}\right) / l\right\}$. From the first step we have $u(t, x)=0$ in a neighbourhood of $[0, \varepsilon] \times K$ for a sufficiently small $\varepsilon>0$. Since $P$ is regularly hyperbolic in $[\varepsilon, T]$ we obtain $u(t, x)=0$ in a neighbourhood of $\overline{D_{l}\left(t_{0}, x_{0}\right)}$. Therefore $u\left(t_{0}, x_{0}\right)=0$. The proof of Theorem 1 is completed.

Q.E.D.

In order to prove Theorem 2 we shall decompose the operator $P$ as follows.

$$
P\left(t, x, D_{t}, D_{x}\right)=Q\left(t, x, D_{t}, D_{x}\right)+R\left(t, x, D_{t}, D_{x}\right)
$$

where

$$
\begin{gathered}
Q=t^{k} D_{t}^{m}+\sum_{j=1}^{k} \sum_{|\beta|=j} a_{j, \beta}(t, x) t^{k-j} D_{t}^{m-j} D_{x}^{\beta}+\sum_{j=h+1}^{m} \sum_{|\beta|=j} a_{j, \beta}(t, x) \\
\times D_{t}^{m-j} D_{x}^{\beta}+\sum_{j=1}^{k} a_{j, 0}(t, x) t^{k-j} D_{t}^{m-j}+\sum_{j=k+1}^{m} a_{j, 0}(t, x) D_{t}^{m-j} \\
R=\sum_{j=2}^{k} \sum_{1 \leqq|\beta| \leqq j-1} a_{j, \beta}(t, x) t^{k-j} D_{t}^{m-j} D_{x}^{\beta} \\
+\sum_{j=k+1}^{m} \sum_{1 \leqq|\beta| \leqq j-1} a_{j, \beta}(t, x) D_{t}^{m-j} D_{x}^{\beta} .
\end{gathered}
$$

We shall demonstrate the existence of a solution by method of successive iteration. Hence we consider the following scheme.

$$
\begin{aligned}
& \left\{\begin{array}{l}
Q u_{0}(t, x)=f(t, x) \\
\left.D_{t}^{i} u_{0}(t, x)\right|_{t=0}=u_{i}(x), \quad 0 \leqq i \leqq m-k-1,
\end{array}\right. \\
& \left\{\begin{array}{l}
Q u_{j}(t, x)=-R u_{j-1}(1, x) \\
\left.D_{t}^{i} u_{j}(t, x)\right|_{t=0}=0, \quad 0 \leqq i \leqq m-k-1, \\
\text { for } j \geqq 1
\end{array}\right.
\end{aligned}
$$

Here we refer Tahara's result [13]. 
Proposition 2.1. Under the assumptions (A-I) $(\mathrm{A}-\mathrm{VI})$ assertions $1^{\circ}$ and $2^{\circ}$ are realized.

$1^{\circ}$ For any $u_{i}(x) \in H^{\infty}\left(\boldsymbol{R}^{n}\right)$ and any $f(t, x) \in \mathscr{B}\left([0, T], H^{\infty}\left(\boldsymbol{R}^{n}\right)\right)$ there exists a unique solution $u(t, x) \in \mathscr{B}\left([0, T], H^{\infty}\left(\boldsymbol{R}^{n}\right)\right)$ of the equation

$$
\left\{\begin{array}{l}
Q\left(t, x, D_{t}, D_{x}\right) u(t, x)=f(t, x) \\
\left.D_{t}^{i} u(t, x)\right|_{t=0}=u_{i}(x), \quad 0 \leqq i \leqq m-k-1 .
\end{array}\right.
$$

$2^{\circ}$ If supp $\left(u_{i}(x)\right) \subset K(0 \leqq i \leqq m-k-1)$ and $\operatorname{supp}(f(t, x)) \subset C_{l}(K)$ hold for any compact set $K \subset \boldsymbol{R}^{\prime}$, then $u(t, x)$ also satisfies supp $(u(t, x)) \subset C_{l}(K)$.

Since $\Gamma^{(\kappa)} \subset H^{\infty}\left(\boldsymbol{R}^{n}\right) u_{0}(t, x)$, solution of $(2.4)_{0}$, belongs to $\mathscr{B}\left([0, T], H^{\infty}\left(\boldsymbol{R}^{n}\right)\right)$ by Proposition 2.1. Noting that $R=R\left(t, x, D_{t}, D_{x}\right)$ is a differential operator, we have $R u_{0}(t, x) \in \mathscr{B}\left([0, T], H^{\propto}\left(\boldsymbol{R}^{n}\right)\right)$. Therefore it follows from $(2.4)_{1}$ and Proposition 2.1 that $u_{1}(t, x)$ also belongs to $\mathscr{B}\left([0, T], H^{\infty}\left(\boldsymbol{R}^{n}\right)\right)$. Successive use of these steps brings us to $u_{j}(t, x) \in \mathscr{B}\left([0, T], H^{\infty}\left(\boldsymbol{R}^{n}\right)\right)$ for any $j \geqq 0$. A formal solution of (1.7) is given in the form

$$
u(t, x)=\sum_{j=0}^{\infty} u_{j}(t, x) .
$$

Accordingly we must show that the summation (2.6) is convergent in some sense.

Our plan is as follows. In $\$ 3$ we shall get an energy inequality of the equation

$$
\left\{\begin{array}{l}
\hat{Q}\left(t, x, D_{t}, D_{x}\right) v(t, x)=g(t, x) \\
\left.D_{t}^{i} v(t, x)\right|_{t=0}=0, \quad 0 \leqq i \leqq s-1
\end{array}\right.
$$

where $\hat{Q}=t^{m-k} Q\left(t, x, D_{t}, D_{x}\right), s$ is a sufficiently large integer and $g(t, x) \in \mathscr{B}([0$, $\left.T], \Gamma^{(\kappa)}\right)$. In $\$ 4$ we shall estimate derivatives of a solution of (2.7) and in $\$ 5$ we obtain estimates of $\hat{R}\left(t, x, D_{t}, D_{x}\right) v(t, x)$ where $\hat{R}=t^{m-h} R\left(t, x, D_{t}, D_{x}\right)$. By the consideration of $\S 4$ and 5 we shall prove Theorem 2 in $\$ 6$.

\section{\$3. Energy Estimates for Solutions of (2.7)}

IFirst we shall define symbol classes of pseudo-differential operators used in this section.

Definition. $1^{\circ}$ For real $m S^{m}$ is the symbol class of classical pseudodifferential operators.

$2^{\circ}$ For positive integer $v$ and real $m \mathscr{B}_{v}\left([0, T], S^{m}\right)$ is the set of functions 
$a(t, x, \xi)$ which are represented in the form

$$
a(t, x, \xi)=\sum_{i=1}^{q} t^{v_{i}} a_{i}(t, x, \xi)
$$

where $v_{i}=v_{i}^{\prime} / v\left(v_{i}^{\prime}\right.$ is a non-negative integer $), q \in N$ and $a_{i}(t, x, \xi) \in \mathscr{B}([0, T]$, $\left.S^{m}\right)$.

The purpose of this section is to show the following lemma.

Lemma 3.1. Let $\Phi(t)$ be

$$
\Phi(t)=\sum_{j=0}^{m-1} \sum_{i=0}^{m-1-j} t^{i l+j}\left\|\Lambda^{i} D_{t}^{j} v\right\|
$$

where $\Lambda$ is the pseudo-differential operator with slimhol $\langle\xi\rangle=\left(1+|\xi|^{2}\right)^{1 / 2}$ Then there exist constants $c_{1}, c_{2}>0$ such that

$$
\Phi(t) \leqq c_{1} \int_{0}^{t} t^{c_{2}} \tau^{-c_{2}-1}\|\hat{Q} v\| d \tau
$$

for $v(t, x) \in \mathscr{B}\left([0, T], H^{\infty}\left(\boldsymbol{R}^{n}\right)\right),\left.\quad D_{t}^{j} v(t, x)\right|_{t=0}=0 \quad 0 \leqq j \leqq s-1$ where $s \geqq N_{1}=$ $c_{2}^{*}+1$ and $c_{2}^{*}$ is the lowest integer greater than or equal to $c_{2}$.

Here we whall note the properties of operator $\widehat{Q}\left(t, x, D_{t}, D_{x}\right)$.

Lemma 3.2. $1^{\circ}$ The partial differential operator $\hat{Q}\left(t, x, D_{t}, D_{x}\right)=$ $t^{m-k} Q\left(t, x, D_{t}, D_{x}\right)$ is decomposed into the sum of $\hat{Q}_{1}\left(t, x, D_{t}, D_{x}\right)$ and $\hat{Q}_{2}(t, x$, $\left.D_{r}, D_{x}\right)$ where

$$
\begin{aligned}
& \hat{Q}_{1}=t^{m} D_{t}^{m}+\sum_{j=1}^{k} \sum_{|\beta|=j} a_{j, \beta}(t, x) t^{m-j} D_{t}^{m-j} D_{x}^{\beta} \\
&+\sum_{j=k+1}^{m} \sum_{|\beta|=j} a_{j, \beta}(t, x) t^{j-k} t^{m-j} D_{t}^{m-j} D_{x}^{\beta}, \\
& \hat{Q}_{2}=\sum_{j=1}^{h} a_{j, 0}(t, x) t^{m-j} D_{t}^{m-j}+\sum_{j=h+1}^{m} a_{j, 0}(t, x) t^{j-k} t^{m-j} D_{t}^{m-j}
\end{aligned}
$$

$2^{\circ}$ The functions $a_{j, \beta}(t, x)(1 \leqq j \leqq k)$ and $t^{j-k} a_{j, \beta}(t, x) \quad(k+1 \leqq j \leqq m)$ are represented by

$$
\begin{aligned}
& a_{j, \beta}(t, x)=t^{l|\beta|} \hat{a}_{j, \beta}(t, x) \quad \text { for } \quad 1 \leqq j \leqq k, \\
& t^{j-k} a_{j, \beta}(t, x)=t^{l|\beta|} \hat{a}_{j, \beta}(t, x) \quad \text { for } \quad k+1 \leqq j \leqq m,
\end{aligned}
$$

where $\hat{a}_{j, \beta}(t, x) \in \mathscr{B}\left([0, T], \gamma^{(\kappa)}\right), 1 \leqq j \leqq m$. $3^{\circ} \hat{Q}_{1}(t, x, \tau, \xi)$ has the following form.

$$
\hat{Q}_{1}(t, x, \tau, \xi)=\prod_{j=1}^{m}\left(t \tau-t^{l} \lambda_{j}(t, x, \xi)\right)
$$


Proof. Multiplying $t^{m-k}$ by $Q$ we can easily obtain $1^{\circ}$ from the equality (2.2). $3^{\circ}$ is a direct conclusion of the assumpsion (A-VI). We have $2^{\circ}$ by expanding the right hand side of $3^{\circ}$.

Following Uryu [15], [16] we shall prove Lemma 3.1. From assumption (A-VI) if $i \neq j, \quad \lambda_{i} \neq \lambda_{j}$ for any $(t, x) \in[0, T] \times \boldsymbol{R}^{n},|\xi|=1$. Here modifying $\lambda_{i}(t, x, \xi)$ near $\xi=0$, we may suppose that for any $i, j(i \neq j)$ there exists a constant $c$ such that

$$
\left|\left(\lambda_{i}-\lambda_{j}\right)(t, x, \xi)\right| \geqq c\langle\xi\rangle
$$

where $\lambda_{i}(t, x, \xi) \in \mathscr{B}\left([0, T], S^{1}\right)$. Furthermore let us note the following. Since $l$ is a positive rational number, $l$ can be written in the form of irreducible fraction $l=v^{\prime} / v, v, v^{\prime} \in N$. Let $\partial_{j}=t D_{t}-t^{l} \lambda_{j}\left(t, x, D_{x}\right)$ where

$$
\lambda_{j}\left(t, x, D_{x}\right) u(t, x)=(2 \pi)^{-n} \int e^{i x \cdot \xi} \lambda_{j}(t, x, \xi) \hat{u}(t, \xi) d \xi .
$$

We define the modules $W_{\mu}(0 \leqq \mu \leqq m-1)$ over the ring of pseudo-differential operators in $x$ of order zero. $\Pi_{m}$ is the operator in the form of $\Pi_{m}=\partial_{1} \partial_{2} \cdots \partial_{m}$. Let $W_{m-1}$ be the module generated by the momomial operators $\Pi_{m} / \partial_{i}=\partial_{1} \partial_{2} \ldots \check{\partial}_{i}$ $\cdots \partial_{m}$ of order $m-1$ and let $W_{m-2}$ be the module generated by the operators $\Pi_{m} / \partial_{i} \partial_{j}(i \neq j)$ and so on.

In order to prove Lemma 3.1 we prepare several lemmas.

Lemma 3.3. For any $i, j$ there exist pseudo-differential operators $A_{i, j}$, $B_{i, j}, C_{i, j} \in \mathscr{B}_{v}\left([0, T], S^{0}\right)$ such that

$$
\left[\partial_{i}, \partial_{j}\right]=A_{i, j} \partial_{i}+B_{i, j} \partial_{j}+C_{i, j}
$$

where $[$,$] is commutator.$

Proof. Let $\sigma_{0}\left(\left[\partial_{i}, \partial_{j}\right]\right)$ be the principal symbol of $\left[\partial_{i}, \partial_{j}\right]$. Then, by the formula of product of pseudo-differential operators, we obtain

$$
\begin{aligned}
\sigma_{0}\left(\left[\partial_{i}, \partial_{j}\right]\right)= & \sum_{\alpha=0}^{n}\left\{D_{\xi_{\alpha}}\left(t \xi_{0}-t^{l} \lambda_{i}\right) \partial_{x_{\alpha}}\left(t \xi_{0}-t^{l} \lambda_{j}\right)\right. \\
& \left.\quad-D_{\xi_{\alpha}}\left(t \xi_{0}-t^{l} \lambda_{j}\right) \partial_{x_{\alpha}}\left(t \xi_{0}-t^{l} \lambda_{i}\right)\right\} \\
= & t^{l} D_{i, j}(t, x, \xi)
\end{aligned}
$$

where $D_{i, j}(t, x, \xi) \in \mathscr{B}_{v}\left([0, T], S^{1}\right)$. Here we used the notations $x_{0}=t, \xi_{0}=\tau$. If we define functions $A_{i, j}(t, x, \xi)$ and $B_{i, j}(t, x, \xi)$ by $A_{i, j}(t, x, \xi)=D_{i, j}(t, x, \xi) /\left(\lambda_{j}-\lambda_{i}\right)$ and $B_{i, j}(t, x, \xi)=D_{i, j}(t, x, \xi) /\left(\lambda_{i}-\lambda_{j}\right)$ respectively, then $A_{i, j}, B_{i, j} \in \mathscr{B}_{v}\left([0, T], S^{0}\right)$ and the equality 


$$
A_{i, j}(t, x, \xi)\left(t \xi_{0}-t^{l} \lambda_{i}\right)+B_{i, j}(t, x, \xi)\left(t \xi_{0}-t^{l} \lambda_{j}\right)=t^{l} D_{i, j}(t, x, \xi)
$$

holds. Then we obtain

$$
\left[\partial_{i}, \partial_{j}\right]=A_{i, j} \partial_{i}+B_{i, j} \partial_{j}+C_{i, j}
$$

for some $C_{i, j}(t, x, \xi) \in \mathscr{B}_{v}\left([0, T], S^{0}\right)$.

Q.E.D.

Lemma 3.4. For any monomial $\omega_{\mu}^{\alpha} \in W_{\mu}(0 \leqq \mu \leqq m-1)$ there exist $\partial_{i}$ and $\omega_{\mu+1}^{\beta} \in W_{\mu+1}$ such that

$$
\partial_{i} \omega_{\mu}^{\alpha}=\omega_{\mu+1}^{\beta}+\sum_{j=1}^{\mu+1} \sum_{\gamma} c_{\gamma, j} \omega_{\mu+1-i}^{\gamma}
$$

where $c_{\gamma, j}(t, x, \xi) \in \mathscr{B}_{v}\left([0, T], S^{0}\right), \omega_{\mu+1-j}^{\gamma} \in W_{\mu+1-j}$.

Proof. For any $\omega_{\mu}^{\alpha}=\partial_{j_{1}} \cdots \partial_{j_{\mu}}\left(j_{1}<j_{2}<\cdots<j_{\mu}\right)$ there exists some $j \notin\left\{j_{1}, \ldots\right.$, $\left.j_{\mu}\right\} 1 \leqq j \leqq m$. Since $\left[\partial_{i}, \partial_{j}\right]=A_{i, j} \partial_{i}+B_{i, j} \partial_{j}+C_{i, j}$ by Lemma 3.3 we have immediately (3.4).

Q.E.D.

Lemma 3.5. Let $\Psi(t)$ be

$$
\Psi(t)=\sum_{j=1}^{m} \sum_{\alpha}\left\|\omega_{m-j}^{\alpha} v\right\| \quad \text { for } \quad v(t, x) \in C^{\infty}\left([0, T] \times \mathbb{R}^{n}\right) .
$$

Then we have the following energy inequality.

$$
t \frac{d}{d t} \Psi(t) \leqq \text { const. } \Psi(t)+\left\|\Pi_{m} v\right\|
$$

Proof. By Lemma 3.4

$$
\partial_{i} \omega_{\mu}^{\alpha} v=\omega_{\mu+1}^{\beta} v+\sum_{j=1}^{\mu+1} \sum_{\gamma} c_{\gamma, j} \omega_{\mu+1-j}^{\gamma} v .
$$

Using $u$ for $\omega_{\mu}^{\alpha} v$ and $g$ for the right hand side of (3.5), we obtain a first order hyperbolic equation $\partial_{i} u=g$. Then

$$
\begin{aligned}
t \frac{d}{d t}\|u\|^{2} & =2 \operatorname{Re}\left(t \frac{d}{d t} u, u\right) \\
& =2 \operatorname{Re}\left(\sqrt{-1} t^{l} \lambda_{i}\left(t, x, D_{x}\right) u+\sqrt{-1} g, u\right) \\
& \leqq \text { const. }\|u\|^{2}+2\|g\| \times\|u\| .
\end{aligned}
$$

Therefore we can easily obtain the following inequality.

$$
t \frac{d}{d t}\left\|\omega_{\mu}^{\alpha} v\right\| \leqq \text { const. }\left\{\left\|\omega_{\mu}^{\alpha} v\right\|+\sum_{j=1}^{\mu+1} \sum_{\gamma}\left\|\omega_{\mu+1-j}^{\gamma} v\right\|\right\}+\left\|\omega_{\mu+1}^{\beta} v\right\|
$$

By the definition of $\Psi(t)$, the desired inequality holds.

Q.E.D.

Lemma 3.6. Under the assumptions of Theorem 2, there exist symbols of 
pseudo-differential operators $c_{\alpha, j}(t, x, \xi) \in \mathscr{B}_{v}\left([0, T], S^{0}\right)$ and monomial operators $\omega_{m-j}^{\alpha} \in W_{m-j}$ such that

$$
\hat{Q}-\Pi_{m}=\sum_{j=1}^{m} \sum_{x} c_{\alpha, j} \omega_{m-j}^{\alpha} .
$$

Proof. We shall show (3.7) by two steps. The first step is to show the following. Let $\Pi_{\mu}=\partial_{i_{1}} \partial_{i_{2}} \cdots \partial_{i_{\mu}}\left(1 \leqq i_{1}<i_{2}<\cdots<i_{\mu} \leqq m\right)$. Then $\sigma\left(\Pi_{\mu}\right)$, the symbol of $\Pi_{\mu}$, can be written in the form:

$$
\sigma\left(\Pi_{\mu}\right)=\prod_{j=1}^{\mu}\left(t \tau-t^{l} \lambda_{i j}\right)+R_{\mu-1}+\cdots+R_{0}
$$

where $R_{\mu-j}(t, x, \tau, \xi)=\sum_{\beta=0}^{\mu-j} b_{\beta, j}(t, x, \xi) t^{l \beta}(t \tau)^{\mu-j-\beta} \quad$ and $\quad b_{\beta, j}(t, x, \xi) \in \mathscr{B}_{v}([0, T]$, $\left.S^{\beta}\right)$.

We carry out the proof of (3.8) by induction on $\mu$. When $\mu=1(3.8)$ is trivial. Assume that (3.8) is valid for $\mu$. Since $\Pi_{\mu+1}=\Pi_{\mu} \partial_{i_{\mu+1}}$ we have the following by the product formula for two pseudo-differential operators.

$$
\sigma\left(\Pi_{\mu+1}\right)=\sigma\left(\Pi_{\mu}\right)\left(t \tau-t^{l} \lambda_{i_{\mu+1}}\right)+\sum_{\alpha \neq 0} D_{\xi}^{\alpha} \sigma\left(\Pi_{\mu}\right) \partial_{x}^{\alpha}\left(t \tau-t^{l} \lambda_{i_{\mu+1}}\right) .
$$

Therefore by the assumption of induction we can easily get (3.8) with $\mu+1$.

The second step is to show (3.7). From (3.8) with $\mu=m$

$$
\sigma\left(\hat{Q}-\Pi_{m}\right)=\sum_{j=1}^{m} \sum_{i=0}^{m-j} c_{i, j}(t, x, \xi) t^{i l}(t \tau)^{m-i-j}
$$

where $c_{i, j}(t, x, \xi) \in \mathscr{B}_{v}\left([0, T], S^{i}\right)$. Let the principal symbol of $\hat{Q}-\Pi_{m}$ be

$$
\widehat{P}_{m-1}(t, x, t \tau, \xi)=\sum_{i=0}^{m-1} c_{i, 1}(t, x, \xi) t^{i l}(t \tau)^{m-1-i} .
$$

We want to determine $A_{j}(t, x, \xi) \in \mathscr{B}_{v}\left([0, T], S^{0}\right)$ so that

$$
\hat{P}_{m-1}(t, x, t \tau, \xi)=\sum_{j=1}^{m-1} A_{j}(t, x, \xi) \prod_{i \neq j}\left(t \tau-t^{l} \lambda_{i}(t, x, \xi)\right) .
$$

Since $\hat{P}_{m-1}\left(t, x, t^{l} \lambda_{j}(t, x, \xi), \xi\right)=t^{l(m-1)} K_{j}(t, x, \xi)$ where $K_{j}(t, x, \xi) \in \mathscr{B}_{v}([0, T]$, $\left.S^{m-1}\right)$, the equality (3.9) gives $t^{l(m-1)} K_{j}(t, x, \xi)=A_{j}(t, x, \xi) t^{l(m-1)} \prod_{i \neq j}\left(\lambda_{j}-\lambda_{i}\right)$.

Then we can find

$$
A_{j}(t, x, \xi)=\left\{\prod_{i \neq j}\left(\lambda_{j}-\lambda_{i}\right)\right\}^{-1} K_{j}(t, x, \check{\zeta})
$$

in $\mathscr{B}_{v}\left([0, T], S^{0}\right)$. Applying (3.8) for $\mu=m-1$, we have

$$
\sigma\left(\widehat{Q}-\Pi_{m}-\sum_{j=1}^{m} A_{j} \prod_{i \neq j} \partial_{i}\right)=\sum_{j=2}^{m} \sum_{i=0}^{m-j} d_{i, j}(t, x, \xi) t^{i l}(t \tau)^{m-i-j}
$$


where $d_{i, j}(t, x, \xi) \in \mathscr{B}_{v}\left([0, T], S^{i}\right)$. Repeating these steps we arrive at the relation (3.7).

Q.E.D.

Lemma 3.7. There exists a constant $c_{1}>0$ such that

$$
\Phi(t) \leqq c_{1} \Psi(t) .
$$

Proof. It is sufficient to show

$$
t^{i 1+j}\left\|\Lambda^{i} D_{t}^{j} v\right\| \leqq \text { const. } \Psi(t) \quad \text { for } \quad 0 \leqq j \leqq m-1,0 \leqq i \leqq m-1-j .
$$

Since the symbol of $t^{i l+j} \Lambda^{i} D_{t}^{j}$ is $t^{i l+j}\langle\xi\rangle^{i} \tau^{j}$, by the same method of the proof of Lemma 3.6 we have

$$
t^{i l+j} \Lambda^{i} D_{t}^{j}=\sum_{i=1}^{m} \sum_{x} d_{x, i} \omega_{m-i}^{\alpha}
$$

where $d_{\alpha, i}(t, x, \xi) \in \mathscr{B}_{v}\left([0, T], S^{0}\right), \omega_{m-i}^{\alpha} \in W_{m-i}$. Hence $t^{i l+j}\left\|\Lambda^{i} D_{t}^{j} v\right\| \leqq$ const. $\times \Psi(t)$.

Q.E.D.

Now we proceed to prove Lemma 3.1. It can be easily seen from Lemma 3.6 that

$$
\begin{aligned}
\left\|\Pi_{m} v\right\| & =\left\|\left(\Pi_{m}-\hat{Q}\right) v+\hat{Q} v\right\| \\
& \leqq\left\|\left(\Pi_{m}-\hat{Q}\right) v\right\|+\|\hat{Q} v\| \\
& \leqq \text { const. } \Psi(t)+\|\hat{Q} v\| .
\end{aligned}
$$

This inequality combined with Lemma 3.5 directly shows

$$
t \frac{d}{d t} \Psi(t) \leqq c_{2} \Psi(t)+\|\hat{Q} v\| \quad \text { for some } \quad c_{2}>0 .
$$

From this inequality

$$
\frac{d}{d t} t^{-c_{2}} \Psi(t) \leqq t^{-c_{2}-1}\|\hat{Q} v\|
$$

Let us note $\left.D_{t}^{j} v(t, x)\right|_{t=0}=0$ for $0 \leqq j \leqq s-1, s \geqq c_{2}^{*}+1$. Therefore we can get the following by integration of both sides of (3.13) from 0 to $t$.

$$
\Psi(t) \leqq \int_{0}^{t} t^{c_{2}} \tau^{-c_{2}-1}\|\hat{Q} v\| d \tau
$$

Finally using Lemma 3.7 we complete the proof of Lemma 3.1. $\quad$ Q.E.D.

\section{§4. Estimate for Solutions of (2.7)}

Assume the existence of solutions of (2.7) 


$$
\left\{\begin{array}{l}
\hat{Q} v(t, x)=g(t, x) \\
\left.D_{t}^{i} v(t, x)\right|_{t=0}=0 \quad \text { for } \quad 0 \leqq i \leqq s-1
\end{array}\right.
$$

where $g(t, x) \in \mathscr{B}\left([0, T], \Gamma^{(\kappa)}\right)$. Then we have

$$
\left.D_{t}^{i} g(t, x)\right|_{t=0}=0 \quad \text { for } \quad 0 \leqq i \leqq s-1 .
$$

Therefore we may assume the following on $g(t, x)$. For any $r \geqq 0$ there exist constants $c, R, K>0$ such that

$$
\left\|\Lambda^{r} g(t, x)\right\| \leqq c R^{r} r !^{\kappa} t^{s} \exp \left(K r^{*} t^{l}\right)
$$

where $r !=\Gamma(r+1)$ and $r^{*}$ is the lowest integer greater than or equal to $r$. For simplification we use the notation $w_{r}(s, t, R)=R^{r} r !^{k} t^{s} \exp \left(K r^{*} t^{l}\right)$.

Now we shall prove the basic lemma of this section.

Lemma 4.1. Let $\Phi_{r}(t)$ be

$$
\Phi_{r}(t)=\sum_{j=0}^{m-1} \sum_{i=0}^{m-1-j} t^{i l+j}\left\|\Lambda^{r+i} D_{t}^{j} v\right\|
$$

Then for any $r \geqq 0$ there exist constants $A, s_{0}>0$ such that for sufficiently large $R, K$ and for $s \geqq s_{0}$

$$
\Phi_{r}(t) \leqq A c s^{-1} w_{r}(s, t, R) .
$$

Proof. We carry out the proof by induction on $r$. When $r=0$, it follows from Lemma 3.1 and (4.2) that for any $s \geqq N_{1}$

$$
\begin{aligned}
\Phi_{0}(t) & \leqq c_{1} \int_{0}^{t} t^{c_{2}} \tau^{-c_{2}-1} c w_{0}(s, \tau, R) d \tau \\
& \leqq c_{1}\left(s-c_{2}\right)^{-1} c w_{0}(s, t, R) .
\end{aligned}
$$

Here we make $s$ sufficiently large such that $s-c_{2} \geqq s / 2$, then we have

$$
\Phi_{0}(t) \leqq A c s^{-1} w_{0}(s, t, R)
$$

where $A \geqq 2 c_{1}, s \geqq s_{0}=\max \left(2 c_{2}, N_{1}\right)$. Assuming that (4.4) is valid for any $0 \leqq r \leqq n$, we shall demonstrate that (4.4) is valid also for $n<r \leqq n+1$. For $r>0$ operating the pseudo-differential operator $\Lambda^{r}$ on both sides of $\hat{Q} v(t, x)=g(t, x)$, we get

$$
\hat{Q} \Lambda^{r} v(t, x)=\Lambda^{r} g(t, x)+\left[\hat{Q}, \Lambda^{r}\right] v(t, x) .
$$

We shall estimate the commutator $\left[\hat{Q}, \Lambda^{r}\right] v(t, x)$. From $1^{\circ}$ and $2^{\circ}$ of Lemma 3.2 


$$
\begin{aligned}
& \hat{Q}_{1}=t^{m} D_{t}^{m}+\sum_{j=1}^{m} \sum_{|\beta|=j} \hat{a}_{j, \beta}(t, x) t^{l|\beta|+m-j} D_{t}^{m-j} D_{x}^{\beta} \\
& \hat{Q}_{2}=\sum_{j=1}^{m} \hat{a}_{j, 0}(t, x) t^{m-j} D_{t}^{m-j} .
\end{aligned}
$$

Therefore

$$
\begin{aligned}
{\left[\hat{Q}, \Lambda^{r}\right]=} & \sum_{j=1}^{m} \sum_{|\beta|=j}\left[\hat{a}_{j, \beta} D_{x}^{\beta}, \Lambda^{r}\right] t^{l|\beta|+m-j} D_{t}^{m-j} \\
& +\sum_{j=1}^{m}\left[\hat{a}_{j, 0}, \Lambda^{r}\right] t^{m-j} D_{t}^{m-j}
\end{aligned}
$$

By use of a product formula of pseudo-differential operators (Lemma A-1 in Appendix) we obtain for $0 \leqq|\beta| \leqq m$

$$
\sigma\left(\left[\hat{a}_{i, \beta} D_{x}^{\beta}, \Lambda^{r}\right]\right)=-\sum_{i=1}^{N-1} g_{i}^{\beta}(t, x, \xi)-r_{N}^{\beta}(t, x, \xi)
$$

where $N=r^{*}+|\beta|$ and

$$
g_{i}^{\beta}(t, x, \xi)=\sum_{|\alpha|=i} \frac{1}{\alpha !} \partial_{\xi}^{\alpha}\langle\xi\rangle^{r} D_{x}^{\alpha} \hat{a}_{j, \beta}(t, x) \xi^{\beta} .
$$

Then from Lemma A-3 there exist constants $\hat{c}, \hat{R}>0$ such that

$$
\begin{aligned}
& \left\|g_{i}^{\beta}\left(t, x, D_{x}\right) u\right\| \leqq \hat{c} \hat{R}^{i-|\beta|}(i-|\beta|) !^{\kappa}\left(\begin{array}{r}
r_{i}^{*} \\
)
\end{array}\right)\left\|\Lambda^{r+|\beta|-i} u\right\| \\
& \text { for } i=1,2, \ldots, r^{*} \text {, } \\
& \left\|g_{i}^{\beta}\left(t, x, D_{x}\right) u\right\| \leqq \hat{c} \hat{R}^{i-|\beta|}(i-|\beta|) !^{k}\left\|\Lambda^{r+|\beta|-i} u\right\| \\
& \text { for } i=r^{*}+1, \ldots, N-1 \text { and } \\
& \left\|r_{N}^{\beta}\left(t, x, D_{x}\right) u\right\| \leqq \hat{c} \hat{R}^{r} r^{\prime \kappa}\|u\| \\
& \text { where } u \in \mathscr{B}\left([0, T], H^{\infty}\left(\mathbb{R}^{n}\right)\right) \text {. }
\end{aligned}
$$

It follows from this estimate that

$$
\begin{aligned}
I_{j, 0}= & \left\|\left[\hat{a}_{j, 0}, \Lambda^{r}\right]\left(t^{m-j} D_{t}^{m-j} v\right)\right\| \\
\leqq & \sum_{i=1}^{r *-1} \hat{c} \hat{R}^{i} i ! \kappa\left(\begin{array}{l}
r^{*} \\
i
\end{array}\right) t^{m-j}\left\|\Lambda^{r-i} D_{t}^{m-j} v\right\| \\
& +\hat{c} \hat{R}^{r} r !^{\kappa} t^{m-j}\left\|D_{t}^{m-j} v\right\|
\end{aligned}
$$

and for $|\beta|=j \geqq 1$

$$
\begin{aligned}
I_{j, \beta}= & \left\|\left[\hat{a}_{j, \beta} D_{x}^{\beta}, \Lambda^{r}\right]\left(t^{l|\beta|+m-j} D_{t}^{m-j} v\right)\right\| \\
\leqq & \sum_{i=1}^{r^{*}} \hat{c} \hat{R}^{i-j}(i-j) ! \kappa\left(\begin{array}{l}
r^{*} \\
i
\end{array}\right) t^{l j+m-j}\left\|\Lambda^{r+j-i} D_{t}^{m-j} v\right\| \\
& +\sum_{i=r^{*}+1}^{j+r^{*-1}} \hat{c} \hat{R}^{i-j}(i-j) !^{\kappa} t^{l j+m-j}\left\|\Lambda^{r+j-i} D_{t}^{m-j} v\right\| \\
& +\hat{c} \hat{R}^{r} r !^{\kappa} t^{l j+m-j}\left\|D_{t}^{m-j} v\right\| .
\end{aligned}
$$


From the assumption of induction we have that

$$
\left\{\begin{array}{c}
t^{l(j-1)+m-j}\left\|\Lambda^{r+j-i} D_{t}^{m-j} v\right\| \leqq A c s^{-1} w_{r-i+1}(s, t, R) \\
\quad \text { for } i=2,3, \ldots, r^{*} \\
t^{l(j-1)+m-j}\left\|\Lambda^{r+j-i} D_{t}^{m-j} v\right\| \leqq A c s^{-1} w_{0}(s, t, R) \\
\quad \text { for } i=r^{*}+1, \ldots, j+r^{*}-1 \\
t^{m-j}\left\|\Lambda^{r-i} D_{t}^{m-j} v\right\| \leqq A c s^{-1} w_{r-i}(s, t, R) \\
\text { for } i=1,2, \ldots, r^{*}-1 \text { and } \\
t^{m-j}\left\|D_{t}^{m-j} v\right\| \leqq A c s^{-1} w_{0}(s, t, R)
\end{array}\right.
$$

Hence it follows from (4.6) that

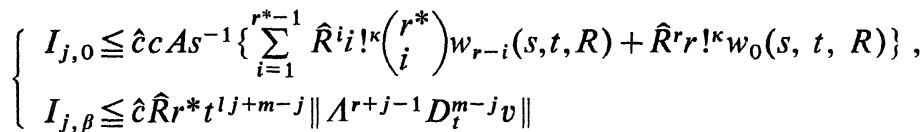

$$
\begin{aligned}
& +\hat{c} c A s^{-1} t^{l}\left\{\sum_{i=2}^{r^{*}} \hat{R}^{i-j}(i-j) !^{\kappa}\left(\begin{array}{l}
r^{*} \\
i
\end{array}\right) w_{r-i+1}(s, t, R)\right. \\
& \left.+\sum_{i=r^{*+1}}^{j+r^{*-1}} \hat{R}^{i-j}(i-j) !^{\kappa} w_{0}(s t, R)+\hat{R}^{r} r !^{\kappa} w_{0}(s, t, R)\right\} .
\end{aligned}
$$

Here let us calculate $I=\sum_{i=1}^{r^{*}-1} \hat{R}^{i} i !^{\kappa}\left(\begin{array}{l}r^{*} \\ i\end{array}\right) w_{r-i}(s, t, R)$.

$$
\begin{aligned}
I & \leqq \sum_{i=1}^{r^{*}-1}(\hat{R} / R)^{i}\left(\begin{array}{l}
r^{*} \\
i
\end{array}\right) i !^{\kappa}(r-i) !^{\kappa} r !^{-\kappa} w_{r}(s, t, R) \\
& =\sum_{i=1}^{r-1}(\hat{R} / R)^{i}\left(\begin{array}{l}
r^{*} \\
i
\end{array}\right)\left(\begin{array}{l}
r \\
i
\end{array}\right)^{-\kappa} w_{r}(s, t, R) .
\end{aligned}
$$

Since $\kappa \geqq 1$ if we make $R \geqq 2 \hat{R}$, then we obtain $I \leqq$ const. $w_{r}(s, t, R)$. Therefore $I_{j, 0} \leqq$ const. $c A s^{-1} w_{r}(s, t, R)$.

Next we shall calculate $J=\sum_{i=2}^{r^{*}} \hat{R}^{i-j}(i-j) ! \kappa\left(\begin{array}{l}r^{*} \\ i\end{array}\right) w_{r-i+1}(s, t, R)$.

$$
\begin{aligned}
J & \leqq \sum_{i=2}^{r^{*}} \hat{R}^{-j} R(\hat{R} / R)^{i}\left(\begin{array}{l}
r^{*} \\
i
\end{array}\right)(i-j) !^{\kappa}(r-i+1) !^{\kappa} r !^{-\kappa} w_{r}(s, t, R) \\
& \leqq r^{*} \sum_{i=2}^{r^{*}} \hat{R}^{-j} R(\hat{R} / R)^{i}\left(\begin{array}{l}
r^{*} \\
i-1
\end{array}\right)\left(\begin{array}{l}
r \\
i-1
\end{array}\right)^{-\kappa} w_{r}(s, t, R) .
\end{aligned}
$$

Let $R \geqq 2 \hat{R}$, then we get $J \leqq$ const. $r^{*} w_{r}(s, t, R)$. Hence the following estimate holds.

$$
\begin{aligned}
I_{j, \beta} \leqq & \hat{c} \hat{R} r^{*} t^{l j+m-j}\left\|A^{r+j-1} D_{t}^{m-j} v\right\| \\
& + \text { const. } c A s^{-1} t^{l} r^{*} w_{r}(s, t, R) .
\end{aligned}
$$

Noting that

$$
\left\|\left[\hat{Q}, \Lambda^{r}\right] v\right\| \leqq \sum_{j=1}^{m}\left\{I_{j, 0}+\sum_{|\beta|=j} I_{j, \beta}\right\}
$$


there exist constants $c_{3}, \tilde{c}>0$ such that

$$
\begin{gathered}
\left\|\left[\hat{Q}, A^{r}\right] v\right\| \leqq \\
c_{3} r^{*} t^{l} \Phi_{r}(t)+\tilde{c} c A s^{-1} r^{*} t^{l} w_{r}(s, t, R) \\
+\tilde{c} c A s^{-1} w_{r}(s, t, R) .
\end{gathered}
$$

By the way, since $\hat{Q} \Lambda^{r} v=\Lambda^{r} g+\left[Q, \Lambda^{r}\right] v$ we can see from Lemma 3.1 that

$$
\Phi_{r}(t) \leqq c_{1} \int_{0}^{t} t^{c_{2}} \tau^{-c_{2}-1}\left\{\left\|\Lambda^{r} g\right\|+\left\|\left[\hat{Q}, \Lambda^{r}\right] v\right\|\right\} d \tau .
$$

Let $f(t)$ be

$$
\begin{aligned}
f(t)= & c_{1} c \int_{0}^{t} t^{c_{2}} \tau^{-c_{2}-1}\left\{w_{r}(s, \tau, R)+\tilde{c} A s^{-1} r^{*} \tau^{l} w_{r}(s, \tau, R)\right. \\
& \left.+\tilde{c} A s^{-1} w_{r}(s, \tau, R)\right\} d \tau
\end{aligned}
$$

then from (4.8)

$$
\Phi_{r}(t) \leqq f(t)+c_{1} c_{3} r^{*} \int_{0}^{t} t^{c_{2}} \tau^{-c_{2}-1+l} \Phi_{r}(\tau) d \tau
$$

Here it follows from Lemma A-4 in appendix that

$$
\Phi_{r}(t) \leqq f(t)+\bar{c} r^{*} \int_{0}^{t} t^{c_{2}} \tau^{-c_{2}-1+l} \exp \left\{\frac{\bar{c} r^{*}}{l}\left(t^{l}-\tau^{l}\right)\right\} f(\tau) d \tau
$$

where $\bar{c}=c_{1} c_{3}$. Noting that $w_{r}(s, 1, R)=R^{r} r^{\prime \kappa} t^{s} \exp \left(K r^{*} t^{l}\right)$, we have

$$
\begin{aligned}
f(t) \leqq & c_{1} c\left\{\left(s-c_{2}\right)^{-1} w_{r}(s, t, R)+\tilde{c} A s^{-1} r^{*}\left(K r^{*} l\right)^{-1} w_{r}(s, t, R)\right. \\
& \left.+\tilde{c} A s^{-1}\left(s-c_{2}\right)^{-1} w_{r}(s, t, R)\right\} .
\end{aligned}
$$

Here we make $K$ and $s$ sufficiently large, then

$$
f(t) \leqq \frac{c A}{2} s^{-1} w_{r}(s, t, R)
$$

Therefore from (4.10)

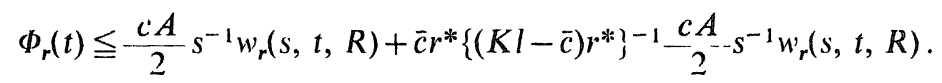

Let $K$ be sufficiently large a number such that $\bar{c}(K l-\bar{c})^{-1} \leqq 1$, then $\Phi_{r}(t) \leqq$ $c A s^{-1} w_{r}(s, t, R)$.

Q.E.D.

Lemma 4.2. For any $r \geqq 0$ and $0 \leqq i+j \leqq m-1$ there exist constants $\bar{A}$, $s_{1}>0$ such that for sufficiently large $R, K$ and for any $s \geqq s_{1}$

$$
t^{i l+j}\left\|\Lambda^{r+i} D_{t}^{j} v\right\| \leqq c \bar{A} s^{-(m-i-j)} w_{r}(s, t, R) .
$$

Proof. It follows from Lemma 4.1 that for any $s \geqq s_{0}$ 


$$
t^{i l+m-1-i}\left\|\Lambda^{r+i} D_{t}^{m-1-i} v\right\| \leqq c \bar{A} s^{-1} w_{r}(s, t, R) .
$$

For any integer $p \geqq 1$ we can see

$$
\|w\| \leqq \int_{0}^{t_{1}} \int_{0}^{t_{2}} \cdots \int_{0}^{t_{p}}\left\|D_{\tau}^{p} w\right\| d \tau d t_{p} d t_{p-1} \cdots d t_{2}
$$

where $t_{1}=t$. Here let $w(t, x)$ and $p$ be $w(t, x)=\Lambda^{r+i} D_{t}^{j} v(t, x)$ and $p=m-1$ $-i-j$ respectively, then we have from (4.12)

$$
\begin{aligned}
\left\|\Lambda^{r+i} D_{t}^{j} v\right\| & \leqq \int_{0}^{t_{1}} \int_{0}^{t_{2}} \cdots \int_{0}^{t_{p}} c A s^{-1} w_{r}\left(s-p^{\prime}, \tau, R\right) d \tau d t_{p} \cdots d t_{2} \\
& \leqq c A s^{-1}(s / 2)^{-p} w_{r}\left(s-p^{\prime}+p, t, R\right)
\end{aligned}
$$

where $p^{\prime}=i l+m-1-i$ and $s$ is sufficiently large such that $s-p^{\prime} \geqq s / 2$. Therefore we conclude that

$$
t^{i l+j}\left\|\Lambda^{r+i} D_{t}^{j} v\right\| \leqq c\left(2^{p} A\right) s^{-(m-i-j)} w_{r}(s, t, R) .
$$

Q.E.D.

Now we shall state the main proposition of this section.

Proposition 4.3. For any integers $1 \leqq j \leqq m-1,0 \leqq i \leqq j, 1 \leqq \hat{k} \leqq j$ and any real $0 \leqq q \leqq 1$ there exist constants $A$ and $s_{1}$ such that

$$
t^{j-i}\left\|\Lambda^{r+i} D_{t}^{j-i} v\right\| \leqq c \bar{A} s^{-(m-j+\hat{k} q)} w_{r+\hat{k} q}(s-i l+\hat{k} q l, t, R)
$$

where $s \geqq s_{1}$ and $R, K$ are sufficiently large numbers.

For the proof of this proposition we need the following lemma of Igari [4].

Lemma 4.4. If $p \geqq 0, q \geqq 0$ and $p+q=1$, then

$$
\left\|\Lambda^{r} u\right\| \leqq\left\|\Lambda^{r-p} u\right\|^{q}\left\|\Lambda^{r+q} u\right\|^{p}
$$

Proof of Proposition 4.3. Let the left hand side of (4.13) be $T_{r}(t)$, then it follows from Lemma 4.2 that

$$
\begin{cases}(4.14)_{1} & T_{r}(t) \leqq c \bar{A} s^{-(m-j+\hat{k})} w_{r+\hat{k}}(s-(i-\hat{k}) l, t, R) \\ (4.14)_{2} & T_{r}(t) \leqq c \bar{A} s^{-(m-j+\hat{k}-1)} w_{r+\hat{k}-1}(s-(i-\hat{k}+1) l, t, R) \\ & \vdots \\ (4.14)_{\hat{k}+1} & T_{r}(t) \leqq c \bar{A} s^{-(m-j)} W_{r}(s-i l, t, R) .\end{cases}
$$

From Lemma 4.4 we immediately have

$$
T_{r}(t) \leqq T_{r-p}(t)^{q} T_{r+q}(t)^{p} .
$$

Combining two inequalities $(4.14)_{1}$ and $(4.14)_{2}$, we can verify that 


$$
T_{r}(t) \leqq c \bar{A} s^{-(m-j+\hat{k}-p)} w_{r+\hat{k}-p}(s-(i-\hat{k}) l-p l, t, R) .
$$

In the same way, combining (4.14) ${ }_{n}$ and $(4.14)_{n+1}$ for $n=2, \ldots, \hat{k}$, we obtain

$$
\begin{aligned}
& T_{r}(t) \leqq c \bar{A} s^{-(m-j+\hat{k}-1-p)} w_{r+\hat{k}-1-p}(s-(i-\hat{k}+1) l-p l, t, R) \\
& \quad \vdots \\
& T_{r}(t) \leqq c \bar{A} s^{-(m-j+1-p)} w_{r+1-p}(s-(i-1) l-p l, t, R) .
\end{aligned}
$$

Next we apply the above process to inequalities $(4.14)_{1}^{\prime}, \ldots,(4.14)_{\hat{k}}^{\prime}$, then

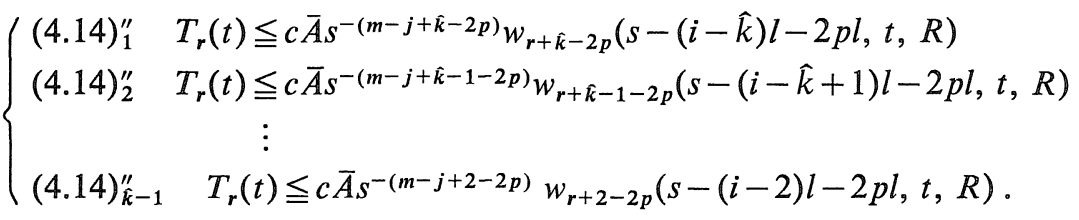

Repeating these steps, we finally attain to the only one inequality as follows.

$$
\begin{aligned}
T_{r}(t) & \leqq c \bar{A} s^{-(m-j+\hat{k}-\hat{k} p)} w_{r+\hat{k}-\hat{k} p}(s-(i-\hat{k}) l-\hat{k} p l, t, R) \\
& =c \bar{A} s^{-(m-j+\hat{k} q)} w_{r+\hat{k} q}(s-i l+\hat{k} q l, t, R) .
\end{aligned}
$$

Q.E.D.

\section{§5. Estimate for $\hat{R} \boldsymbol{p}$}

We begin with the following lemma.

Lemma 5.1. The partial differential operator $\widehat{R}=t^{m-k} R\left(t, x, D_{t}, D_{x}\right)$ is represented in the form:

$$
\hat{R}\left(t, x, D_{t}, D_{x}\right)=\sum_{j=1}^{m-1} \sum_{|\beta|=1}^{j} \hat{a}_{m+|\beta|-j, \beta}(t, x) t^{\alpha(j, \beta)+j-|\beta|} D_{t}^{j-|\beta|} D_{x}^{\beta} .
$$

Proof. From (2.3), (1.5) and (1.6) we have

$$
\begin{aligned}
\widehat{R}= & \sum_{j=2}^{k} \sum_{|\beta|=1}^{j-1} \hat{a}_{j, \beta}(t, x) t^{\gamma(j, \beta)+m-j} D_{t}^{m-j} D_{x}^{\beta} \\
& +\sum_{j=k+1}^{m} \sum_{|\beta|=1}^{j-1} \hat{a}_{j, \beta}(t, x) t^{\gamma(j, \beta)+j-k+m-j} D_{t}^{m-j} D_{x}^{\beta} \\
= & \sum_{j=2}^{m} \sum_{|\beta|=1}^{j-1} \hat{a}_{j, \beta}(t, x) t^{\alpha(m-j+|\beta|, \beta)+m-j} D_{t}^{m-j} D_{x}^{\beta} .
\end{aligned}
$$

Let us replace $m-j+|\beta|$ with $j$. Hence we can get the desired result. Q.E.D.

It follows from Lemma 4.3 that for any integer $1 \leqq k_{j} \leqq j$ and real $0 \leqq q \leqq 1$

$$
\begin{aligned}
& t^{\alpha(j, \beta)+j-|\beta|}\left\|\Lambda^{r} D_{t}^{j-|\beta|} D_{x}^{\beta} v\right\| \leqq c \bar{A} s^{-\left(m-j+k_{j} q\right)} \\
& \quad \times w_{r+k_{j} q}\left(s-|\beta| l+k_{j} q l+\alpha(j, \beta), t, R\right)
\end{aligned}
$$


Then owing to Lemma 5.1, we have

$$
\begin{aligned}
\left\|\Lambda^{r} \hat{R} v\right\| \leqq & c \bar{A} B \sum_{j=1}^{m-1} \sum_{|\beta|=1}^{j} s^{-\left(m-j+k_{j} q\right)} \\
& \times w_{r+k_{j} q}\left(s-|\beta| l+k_{j} q l+\alpha(j, \beta), t, R\right)
\end{aligned}
$$

where $B>0$ is independent of $r$. Here let $1 \leqq k_{j} \leqq j$ be the smallest integer satisfying the inequalities:

$$
\alpha(j, \beta) / l-|\beta|+k_{j}>0 \quad \text { for any } \quad \beta(1 \leqq|\beta| \leqq j-1) .
$$

Next we shall remember the definition of $\sigma_{j}$ and $v$.

$$
\sigma_{j}=\max _{1 \leqq|\beta| \leqq j-1}\{|\beta|-\alpha(j, \beta) / l, 0\}, \quad v=\max _{1 \leqq j \leqq m-1}\left\{\sigma_{j} / k_{j}\right\} .
$$

Then we can verify that

$$
-|\beta| l+k_{j} q l+\alpha(j, \beta) \geqq(q-v) l .
$$

Since $0 \leqq v<1$ we make $0 \leqq q \leqq 1$ satisfying $q>v$. Hence it follows from (5.1) and (5.3) that

$$
\left\|\Lambda^{r} \hat{R} v\right\| \leqq c \bar{A} B \sum_{j=1-1}^{m-1} s^{-\left(m-j+k_{J} q\right)} w_{r+k_{j} q}(s+(q-v) l, t, R) .
$$

Furthermore let $\theta$ be a positive number so that for $1 \leqq j \leqq m-1$

$$
k_{j} q \theta \leqq m-j+k_{j} q
$$

then

$$
\left\|\Lambda^{r} \hat{R} v\right\| \leqq c \bar{A} B \sum_{j=1}^{m-1} s^{-k_{\jmath} q \theta} W_{r+k_{j} q}(s+(q-v) l, t, R) .
$$

Let us summarize the above results.

Lemma 5.2. Let $v(t, x)$ be the solution of the equation

$$
\left\{\begin{array}{l}
\hat{Q} v(t, x)=g(t, x) \\
\left.D_{t}^{i} v(t, x)\right|_{t=0}=0 \quad 0 \leqq i \leqq s-1
\end{array}\right.
$$

where $s \geqq s_{1}$ and $g(t, x)$ satisfies the following inequality.

$$
\left\|\Lambda^{r} g\right\| \leqq c w_{r}(s, t, R) .
$$

Then there exist constants $\bar{A}, B>0$ which are independent of $r$ such that for sufficiently large $R$ and $K$

$$
\left\|A^{r} v\right\| \leqq c \bar{A} s^{-m} w_{r}(s, t, R) \leqq c \bar{A} w_{r}(s, t, R),
$$




$$
\left\|\Lambda^{r} \hat{R} v\right\| \leqq c \bar{A} B \sum_{j=1}^{m-1} s^{-k_{j} q \theta} w_{r+k_{j} q}(s+(q-v) l, t, R),
$$

where $q>v, 0 \leqq q \leqq 1$ and $\theta(>0)$ satisfies the property that for $1 \leqq j \leqq m-1$ $k_{j} q \theta \leqq m-j+k_{j} q$.

\section{§6. Proof of Theorem 2}

We shall first prepare the following basic lemma.

Lemma 6.1. Under the assumptions (A-I) (A-VI), the assertions $1^{\circ}$ and $2^{\circ}$ hold.

$1^{\circ}$ For any $u^{i}(x) \in \Gamma^{(\kappa)}$ and any $\hat{f}(t, x) \in \mathscr{B}\left([0, T], \Gamma^{(\kappa)}\right)$ satisfying $\left.D_{t}^{i} \hat{f}(t, x)\right|_{t=0}=0(0 \leqq i \leqq m-k-1)$ there exists a unique solution $u(t, x) \in \mathscr{B}([0$, $\left.T], \Gamma^{(\kappa)}\right)$ of the equation

$$
\left\{\begin{array}{l}
\hat{Q}\left(t, x, D_{t}, D_{x}\right) u(t, x)=\hat{f}(t, x) \\
\left.D_{t}^{i} u(t, x)\right|_{t=0}=u^{i}(x), \quad 0 \leqq i \leqq m-k-1 .
\end{array}\right.
$$

$2^{\circ} \quad$ Especially if $u^{i}(x) \equiv 0$ for $0 \leqq i \leqq m-k-1$ and $\left.D_{t}^{i} \hat{f}(t, x)\right|_{t=0}=0$ for $0 \leqq i$ $\leqq m-k-1+\hat{s}$ we obtain that $\left.D_{t}^{i} u(t, x)\right|_{t=0}=0$ for $0 \leqq i \leqq m-k-1+\hat{s}$ where $\hat{s}$ is a non-negative integer.

Proof. 1" From the assumption $\hat{f}(t, x)=t^{m-k} h(t, x)$ wherc $h(t, x)$ belongs to $\mathscr{B}\left([0, T], \Gamma^{\left({ }^{n}\right)}\right)$. Therefore we have the equation equivalent to $(6.1)$

$$
\left\{\begin{array}{l}
Q\left(t, x, D_{t}, D_{x}\right) u(t, x)=h(t, x) \\
\left.D_{t}^{i} u(t, x)\right|_{t=0}=u^{i}(x), \quad 0 \leqq i \leqq m-k-1 .
\end{array}\right.
$$

Applying Proposition 2.1, we know the unique existence of the solution $u(t, x) \in$ $\mathscr{B}\left([0, T], H^{\mathcal{X}}\left(\boldsymbol{R}^{\prime \prime}\right)\right)$ of $(6.2)$. Hence let us show that $u(t, x)$ belongs to $\mathscr{B}([0, T]$, $\left.\Gamma^{\left({ }^{\prime}\right)}\right)$. It follows from (A-IV) that we can calculate the derivatives of $u(t, x)$ at $t=0$ and each derivative belongs to $\Gamma^{(h)}$. Here for any integer $s \geqq 1$ let $u_{s}(t, x)$ be

$$
u_{s}(t, x)=u(t, x)-\sum_{i=0}^{s-1} t^{i} /\left.i ! \partial_{t}^{i} u(t, x)\right|_{t-0},
$$

then $u_{s}(1, x)$ satisfics the equation

$$
\hat{Q} u_{s}(t, x)=\hat{f}(t, x)-\hat{Q}\left(\sum_{i=1}^{s-1} t^{i} /\left.i ! \partial_{t}^{i} u(t, x)\right|_{t=0}\right) \equiv f_{s}(t, x) .
$$

Hence we have that $f_{s}(t, x) \in \mathscr{B}\left([0, T], \Gamma^{(\kappa)}\right)$ and 


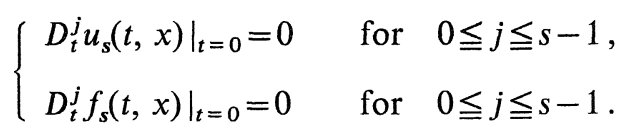

We can reduce (6.1) to the equation

$$
\left\{\begin{array}{l}
\hat{Q} u_{s}(t, x)=f_{s}(t, x) \\
\left.D_{t}^{j} u_{s}(t, x)\right|_{t=0}=0 \quad \text { for } 0 \leqq j \leqq s-1 .
\end{array}\right.
$$

From Lemma 5.2 we can see that $u_{s}(t, x) \in \mathscr{B}\left([0, T], \Gamma^{(\kappa)}\right)$. Consequently $u(t, x)$ belongs to $\mathscr{B}\left([0, T], \Gamma^{(\kappa)}\right)$. Assertion $2^{\circ}$ is clear from (A-IV).

Q.E. D.

Let us consider the scheme $(2.4)_{j}$. Then $(2.4)_{j}$ is equivalent to the following scheme.

$$
\begin{aligned}
& \left\{\begin{array}{l}
\hat{Q} u_{0}(t, x)=t^{m-k} f(t, x) \\
\left.D_{t}^{i} u_{0}(t, x)\right|_{t=0}=u^{i}(x), \quad 0 \leqq i \leqq m-k-1,
\end{array}\right. \\
& \left\{\begin{array}{l}
\hat{Q} u_{j}(t, x)=-\hat{R} u_{j-1}(t, x) \equiv f_{j-1}(t, x) \\
\left.D_{t}^{i} u_{j}(t, x)\right|_{t=0}=0, \quad 0 \leqq i \leqq m-k-1
\end{array} \text { for } j \geqq 1 .\right.
\end{aligned}
$$

Lemma 6.2. Let $u_{j}(t, x)$ be the solution of $(6.4)_{j}$, then $u_{j}(t, x) \in \mathscr{B}([0$, $\left.T], \Gamma^{(\kappa)}\right)$ for $j \geqq 0$ and there exists an integer $\tilde{s} \geqq 1$ such that for $j \geqq 1$ $\left.D_{t}^{i} u_{j}(t, x)\right|_{t=0}=0$ for $0 \leqq i \leqq m-k-1+\tilde{s}(j-1)$.

Proof. From Lemma 6.1 we have $u_{0}(t, x) \in \mathscr{B}\left([0, T], \Gamma^{(\kappa)}\right)$. Since $\hat{R}=$ $t^{m-k} R\left(t, x, D_{t}, D_{x}\right), f_{0}(t, x)$ satisfies that $f_{0}(t, x) \in \mathscr{B}\left([0, T], \Gamma^{(\kappa)}\right)$ and for $0 \leqq i$ $\leqq m-k-\left.1 D_{t}^{i} f_{0}(t, x)\right|_{t=0}=0$. Therefore it follows from Lemma 6.1 that $u_{1}(t$, $x) \in \mathscr{B}\left([0, T], \Gamma^{(\kappa)}\right)$. Repeating these steps, $u_{j}(t, x) \in \mathscr{B}\left([0, T], \Gamma^{(\kappa)}\right)$ for $j \geqq 0$.

Let us consider the second assertion. From $2^{\circ}$ of Lemma 6.1 we can verify that $\left.D_{t}^{i} u_{1}(t, x)\right|_{t=0}=0$ for $0 \leqq i \leqq m-k-1$. Put $\tilde{s}=\min \{\alpha(j, \beta) ; 1 \leqq j \leqq m-1$, $1 \leqq|\beta| \leqq j\} \geqq 1$. Then it follows from Lemma 5.1 that $\left.D_{t}^{i} f_{1}(t, x)\right|_{t=0}=0$ for $0 \leqq i \leqq m-k-1+\tilde{s}$. By Lemma 6.1 we obtain $\left.D_{t}^{i} u_{2}(t, x)\right|_{t=0}=0$ for $0 \leqq i$ $\leqq m-k-1+\tilde{s}$. Successive use of these steps brings us to

$$
\left.D_{t}^{i} u_{j}(t, x)\right|_{t=0}=0 \quad \text { for } \quad 0 \leqq i \leqq m-k-1+\tilde{s}(j-1) .
$$

The following lemma is the direct consequence of Lemma 6.2.

Lemma 6.3. For any $s \geqq 0$ there exists $N=N(s) \in \mathbb{Z}$ such that for any $j \geqq N-\left.1 D_{t}^{i} u_{j}(t, x)\right|_{t=0}=0,0 \leqq i \leqq s-1$. 
Now let us demonstrate the proof of Theorem 2. By Lemma 6.2 and Lemma 6.3 we may assume that for any $r \geqq 0$

$$
\left\|\Lambda^{r} \hat{R} u_{N-1}\right\| \leqq c w_{r}(s, t, R)
$$

where $c$ and $R$ are positive constants. Hence the following lemma holds.

Lemma 6.4. Under (6.5) if $\kappa<\theta$ there exist constants $\tilde{A}, \widetilde{B}, \widetilde{R}$ which are independent of $r$ such that

$$
\left\|\Lambda^{r} u_{N+n}\right\| \leqq c \widetilde{B} \tilde{A}^{n} n^{n(\kappa-\theta)} q_{w_{r}}(s+n(q-v) l, t, \widetilde{R})
$$

for $n=0,1, \ldots$, where $q, v, \theta$ are the same as in Lemma 5.2 .

Proof. It follows from Lemma 5.2 and (6.5) that

$$
\left\{\begin{array}{l}
\left\|\Lambda^{r} u_{N}\right\| \leqq c \bar{A} w_{r}(s, t, R) \\
\left\|\Lambda^{r} \hat{R} u_{N}\right\| \leqq c \bar{A} B \sum_{j=1}^{m-1} s^{-k_{J} q \theta} w_{r+k_{J} q}(s+(q-v) l, t, R) .
\end{array}\right.
$$

Next applying Lemma 5.2 to $(6.4)_{N+1}$,

$$
\left\{\begin{aligned}
\left\|\Lambda^{r} u_{N+1}\right\| \leqq & \bar{A}^{2} B \sum_{j=1}^{m-1} s^{-k_{j} q \theta} w_{r+k_{j} q}(s+(q-v) l, t, R) \\
\left\|\Lambda^{r} \hat{R} u_{N+1}\right\| \leqq & c \bar{A}^{2} B^{2} \sum_{i, j=1}^{m-1}\{s+(q-v) l\}^{-k_{i} q \theta} s^{-k_{j} q \theta} \\
& \times w_{r+\left(k_{i}+k_{j}\right) q}(s+2(q-v) l, t, R) .
\end{aligned}\right.
$$

Inductively we obtain that for any $n \geqq 0$

$$
\begin{aligned}
\left\|\Lambda^{r} u_{N+n}\right\| \leqq c \bar{A}^{n+1} B^{n} \sum_{i_{1}=1}^{m-1} & \ldots \sum_{i_{n}=1}^{m-1} e_{k_{i_{1}}, \ldots, k_{t_{n}}} \\
& \times w_{r+\left(k_{i_{1}}+\cdots+k_{i_{n}}\right) q}(s+n(q-v) l, t, R)
\end{aligned}
$$

where $e_{k_{i_{1}}, \ldots, k_{i_{n}}}=\{s+(q-v) l(n-1)\}^{-k_{i_{n}} q \theta} \times \cdots \times s^{-k_{i_{1}} q \theta}$.

Let us make $s$ sufficiently large, then $e_{k_{t_{1}}, \ldots, k_{t_{n}}}$ is estimated as follows.

$$
e_{h_{11}, \ldots, h_{h_{n}}} \leqq D^{n} n^{-h_{h_{n}} q \theta}(n-1)^{-h_{\iota_{n-1}} q \theta} \times \cdots \times 1^{-h_{\iota_{1}} q \theta}
$$

for some constant $D>0$. Furthermore from Lemma A-5 in Appendix

$$
e_{k_{i_{1}}, \ldots, h_{i_{n}}} \leqq A_{1} R_{1}^{n} D^{n} n^{-\left(k_{i_{1}}+\cdots+k_{i_{n}}\right) q \theta}
$$

Using Lemma A-7, the estimates (6.7) and (6.8) imply that

$$
\begin{aligned}
\left\|\Lambda^{r} u_{N+n}\right\| & \leqq c A_{1} A_{3} \bar{A}^{n+1} B^{n} D^{n} R_{1}^{n} R_{3}^{n} \\
& \times \sum_{i_{1}=1}^{m-1} \ldots \sum_{i_{n}=1}^{m-1} n^{\left(k_{i_{1}}+\cdots+k_{n}\right)(\kappa-\theta) q_{2}} w_{r}\left(s+n(q-v) l, t, R^{\prime}\right) .
\end{aligned}
$$

Here let us make $\kappa<\theta$, then it follows from the above inequality that 


$$
\left\|\Lambda^{r} u_{N+n}\right\| \leqq c \widetilde{B} \tilde{A}^{n} n^{(\kappa-\theta) n q} w_{r}(s+n(q-v) l, t, \tilde{R})
$$

for some constants $\tilde{A}, \widetilde{B}, \widetilde{R}$.

Q.E.D.

Now we shall prove the convergence of summation $\sum_{j=0}^{\infty} u_{j}(t, x)$.

Lemma 6.5. If $\kappa$ satisfies $1 \leqq \kappa<\sigma /(\sigma-1)$, the series $u(t, x)=\sum_{j=0}^{\infty} u_{j}(t, x)$ is convergent in $\mathscr{B}\left([0, T], \Gamma^{(\kappa)}\right)$. Hence $u(t, x)$ belongs to $\mathscr{B}\left([0, T], \Gamma^{(\kappa)}\right)$.

Proof. First of all we shall show that if $1 \leqq \kappa<\sigma /(\sigma-1)$ there exist constants $q, \theta$ satisfying $(6.9) \sim(6.11)$.

$$
\begin{aligned}
& q>v, 0 \leqq q \leqq 1 \\
& \kappa<0
\end{aligned}
$$

$$
\text { For any } 1 \leqq j \leqq m-1, k_{j} q \theta \leqq m-j+k_{j} q .
$$

If $1 \leqq \kappa<\sigma /(\sigma-1)$ we have

$$
\kappa<\sigma /(\sigma-1) \leqq\left(k_{j} v+m-j\right) / k_{j} v \quad\left(\equiv k_{j, v}\right) \quad \text { for } \quad 1 \leqq j \leqq m-1 .
$$

Then for any $\kappa<k_{j, v}$ there exist constants $q>v, \theta>\kappa$ so as $\theta \leqq k_{j, q}$. These $q$ and $\theta$ satisfy (6.9) (6.11). Therefore we can apply Lemma 6.4. Here let us decompose $u(t, x)$ by

$$
\begin{aligned}
u(t . x) & =\sum_{i=0}^{N-1} u_{j}(t, x)+\sum_{j=N}^{\infty} u_{j}(t, x) \\
& =u_{N}^{1}(t, x)+u_{N}^{2}(t, x) .
\end{aligned}
$$

From Lemma 6.4 the series $u_{N}^{2}(t, x)$ is convergent in $\mathscr{B}\left([0, T], \Gamma^{(\kappa)}\right)$ and $u_{N}^{2}(t, x)$ belongs to $\mathscr{B}\left([0, T], \Gamma^{(\kappa)}\right)$. Since $u_{j}(t, x) \in \mathscr{B}\left([0, T], \Gamma^{(\kappa)}\right)$ for $1 \leqq j \leqq N-1$ we can see $u(t, x) \in \mathscr{B}\left([0, T], \Gamma^{(\kappa)}\right)$.

Q.E.D.

Consequently we have established the existence of a solution in Theorem 2. Next we shall show the uniqueness of solutions.

Lemma 6.6. Under the assumptions of Theorem 2, let $u(t, x) \in \mathscr{B}([0$, $\left.T], \Gamma^{(\kappa)}\right)$ be a solution of a homogeneous equation;

$$
\left\{\begin{array}{l}
P\left(t, x, D_{t}, D_{x}\right) u(t, x)=0 \\
\left.D_{t}^{i} u(t, x)\right|_{t=0}=0 \quad \text { for } 0 \leqq i \leqq m-k-1
\end{array}\right.
$$

where $1 \leqq \kappa<\sigma /(\sigma-1)$. Then $u(t, x)$ vanishes identically.

Proof. From the assumption (A-VI) $u(t, x)$ is flat at $t=0$. Hence $u(t, x)$ satisfies the following. 


$$
\left\{\begin{array}{l}
\hat{Q} u(t, x)=-\hat{R} u(t, x) \\
\left.D_{t}^{i} u(t, x)\right|_{t=0}=0 \quad \text { for any } \quad i \geqq 0 .
\end{array}\right.
$$

We may assume that for sufficiently large $s$ there exist constants $c$ and $R$ such that

$$
\left\|\Lambda^{r} u\right\| \leqq c w_{r}(s, t, R) \quad \text { for any } r \geqq 0 .
$$

By Lemma 6.4 we can get from the above estimate that

$$
\left\|\Lambda^{r} u\right\| \leqq c \widetilde{B} \widetilde{A}^{n} n^{n(\kappa-\theta) q_{w}} w_{r}(s+n(q-v) l, t, \widetilde{R}) .
$$

Let $n$ be infinity, then we conclude $u(t, x) \equiv 0$.

Q.E.D.

Finally we shall demonstrate that $2^{\prime \prime}$ of Theorem 2 is realized by convergence of (2.6) and $2^{\circ}$ in Proposition 2.1. From Proposition 2.1 and $(2.4)_{0}$ if supp $\left(u^{i}(x)\right) \subset K$ and $\operatorname{supp}(f(t, x)) \subset C_{l}(K)$ for some compact set $K \subset \mathbb{R}^{n}, \operatorname{supp}\left(u_{0}(t, x)\right)$ $\subset C_{l}(K)$. Since $R\left(t, x, D_{t}, D_{x}\right)$ is a differential operator, we get $\operatorname{supp}\left(R u_{0}(t, x)\right)$ $\subset C_{l}(K)$. Therefore it follows from $(2.4)_{1}$ and Proposition 2.1 that supp $\left(u_{1}(t, x)\right)$ belongs to $C_{l}(K)$. Repeating these steps we have supp $\left(u_{j}(t, x)\right)$ $\subset C_{l}(K)$ for any $j \geqq 0$. From the convergence of $(2.6)$ we conclude supp $(u(t, x))$ $\subset C_{I}(K)$. The proof of Theorem 2 is completed.

\section{Appendix}

Following Igari [4], we introduce a certain class of pseudo-differential operators.

Definition. 1) For any $m \in \mathbb{R}^{1}$ and $\kappa>1$ we denote $b y S^{m}(\kappa)$ the set of functions $h(x, \xi) \in C^{\infty}\left(\boldsymbol{R}^{n} \times \boldsymbol{R}^{n}\right)$ satisfying the property that for any multiindices $\alpha, \beta$, there exist constants $c_{\alpha}$ and $R$ such that

$$
\left|\partial_{\xi}^{\alpha} D_{x}^{\beta} h(x, \xi)\right| \leqq c_{\alpha} R^{|\beta|}(|\beta| !)^{\kappa}\langle\xi\rangle^{m-|\alpha|} \quad \text { for } \quad(x, \xi) \in \mathbb{R}^{n} \times \mathbb{R}^{n} .
$$

2) For any $h(x, \xi) \in S^{m}(\kappa)$ we shall define semi-norms of $h(x, \xi)$ such that for any integer $l \geqq 0$

$$
|h(x, \xi)|_{l}=\max _{|\alpha+\beta| \leqq l} \sup _{(x, \xi) \in R^{n \times R^{n}}}\left|\partial_{\xi}^{\alpha} D_{x}^{\beta} h(x, \xi)\right|\langle\xi\rangle^{-m+|\alpha|} .
$$

We define a pseudo-differential operator with a symbol $h(x, \xi) \in S^{m}(\kappa)$ by

$$
H\left(x, D_{x}\right) u=(2 \pi)^{-n} \int e^{i x \cdot \xi} h(x, \xi) \hat{u}(\xi) d \xi
$$


Hence we have a composition formula of pseudo-differential operators.

Lemma A-1. (See Igari (4)). Let $h(x, \xi) \in S^{m}(\kappa)$ and $r \geqq 0$. Then

$$
\sigma\left(\Lambda^{r} H\right)=\sum_{i=1}^{N-1} \sum_{|\gamma|=i} 1 / \gamma ! \partial_{\xi}^{\gamma}\langle\xi\rangle^{r} h_{\gamma}(x, \xi)+r_{N}(x, \xi)
$$

where $N=r^{*}+m$ and $h_{y}(x, \xi)=D_{x}^{y} h(x, \xi)$. Then for any integer $l \geqq 0$ there exist constants $c_{l}, R>0$ such that

$$
\begin{gathered}
\left|h_{\gamma}(x, \xi)\langle\xi\rangle^{-m}\right|_{l} \leqq c_{l} R^{|\gamma|-m}(|\gamma|-m) !^{\kappa} \\
\left|r_{N}(x, \xi)\right|_{l} \leqq c_{l} R^{r} r !^{\kappa} .
\end{gathered}
$$

The following lemma is well known.

Lemma A-2. For any $h(x, \xi) \in S^{0}$ there exists constant $c$ and nonnegative integer $l$ dependent only on dimension $n$ such that

$$
\left\|H\left(x, D_{x}\right) u\right\| \leqq c|h(x, \xi)|_{\imath}\|u\| .
$$

Lemma A-3. Under the assumptions of Lemma A-1 we denote $h_{i}(x, \xi)$ by

$$
h_{i}(x, \xi)=\sum_{|\gamma|=i} 1 / \gamma ! \partial_{\xi}^{\gamma}\langle\xi\rangle^{r} h_{\gamma}(x, \xi) .
$$

Then there exist $\hat{c}, \hat{R}>0$ such that

$$
\left\{\begin{array}{l}
\left\|H_{i}\left(x, D_{x}\right) u\right\| \leqq \hat{c} \hat{R}^{i-m}(i-m) !^{\kappa}\left(\begin{array}{l}
r^{*} \\
i
\end{array}\right)\left\|\Lambda^{m+r-i} u\right\| \quad \text { for } 1 \leqq i \leqq r^{*} \\
\left\|H_{i}\left(x, D_{x}\right) u\right\| \leqq \hat{c} \hat{R}^{i-m}(i-m) !^{\kappa}\left\|\Lambda^{m+r-i} u\right\| \quad \text { for } \quad r^{*}+1 \leqq i \leqq N-1,
\end{array}\right.
$$

(A.5) $\quad\left\|R_{N}\left(x, D_{x}\right) u\right\| \leqq \hat{c} \hat{R}^{r} r !^{\kappa}\|u\|$.

Proof. (A.5) is a direct consequence of (A.2) and Lemma A-2. For the proof of (A.4) it is sufficient to show the following inequalities.

$$
\left\{\begin{array}{l}
\left|h_{i}(x, \xi)\langle\xi\rangle^{-m-r+i}\right|_{l} \leqq \tilde{c} \hat{R}^{i-m}(i-m) !^{\kappa}\left(\begin{array}{l}
r^{*} \\
i
\end{array}\right) \text { for } 1 \leqq i \leqq r^{*} \\
\left|h_{i}(x, \xi)\langle\xi\rangle^{-m-r+i}\right|_{l} \leqq \tilde{c} \hat{R}^{i-m}(i-m) !^{\kappa} \quad \text { for } \quad r^{*}+1 \leqq i \leqq N-1
\end{array}\right.
$$

for some constant $\tilde{c}$.

We can easily see that for any $\left|\alpha^{\prime}\right| \leqq l$

(A.7) $\sum_{|\gamma|=i} 1 / \gamma !\left|\partial_{\xi}^{\alpha^{\prime}}\left\{\langle\xi\rangle^{-r+i} \partial_{\xi}^{\gamma}\langle\xi\rangle^{r}\right\}\right| \leqq\left\{\begin{array}{l}A^{i}\left(\begin{array}{l}r^{*} \\ i\end{array}\right)\langle\xi\rangle^{-\left|\alpha^{\prime}\right|} \text { for } 1 \leqq i \leqq r^{*}, \\ A^{i}\langle\xi\rangle^{-\left|\alpha^{\prime}\right|} \text { for } r^{*}+1 \leqq i \leqq N-1,\end{array}\right.$ where $A$ is independent of $r$ and $i$. 
Now we shall estimate the absolute value of

$$
\begin{aligned}
I_{\alpha, \beta}= & \partial_{\xi}^{\alpha} D_{x}^{\beta}\left\{h_{i}(x, \xi)\langle\xi\rangle^{-m-r+i}\right\} \quad \text { for }|\alpha+\beta| \leqq l . \\
\left|I_{\alpha, \beta}\right|= & \mid \sum_{|\gamma|=i \alpha^{\prime} \leqq \alpha} 1 / \gamma !\left(\begin{array}{l}
\alpha \\
\alpha^{\prime}
\end{array}\right) \partial_{\xi}^{\alpha^{\prime}}\left\{\langle\xi\rangle^{-r+i} \partial_{\xi}^{\gamma}\langle\xi\rangle^{r}\right\} \\
& \times \partial_{\xi}^{\alpha-\alpha^{\prime}} D_{x}^{\beta}\left\{\langle\xi\rangle^{-m} h_{\gamma}(x, \xi)\right\} \mid \\
\leqq & \sum_{\alpha^{\prime} \leqq \alpha}\left(\begin{array}{l}
\alpha \\
\alpha^{\prime}
\end{array}\right) \sum_{|\gamma|=i} 1 / \gamma ! \mid \partial_{\xi}^{\alpha^{\prime}}\left\{\langle\xi\rangle^{-r+i} \partial \gamma_{\xi}\langle\xi\rangle^{r}\right\} \\
& \times\left|h_{\gamma}(x, \xi)\langle\xi\rangle^{-m}\right|_{l}\langle\xi\rangle^{-\left|\alpha-\alpha^{\prime}\right|} .
\end{aligned}
$$

It follows from (A.1) and (A.7)

$$
\left|I_{\alpha, \beta}\right| \leqq\left\{\begin{array}{l}
B A^{i} R^{i-m}\left(\begin{array}{l}
r^{*} \\
i
\end{array}\right)(i-m) !^{\kappa}\langle\xi\rangle^{-|\alpha|} \quad i=1,2, \ldots, r^{*} \\
B A^{i} R^{i-m}(i-m) !^{\kappa}\langle\zeta\rangle^{-|\alpha|} \quad i=r^{*}+1, \ldots, N-1
\end{array}\right.
$$

for some constant $B$. The proof is completed.

Q.E.D.

Lemma $\mathbb{A}-4$. Let $\phi(t)$ and $\psi(t) \in C^{0}([0, T])$. Assume that the following integral inequality is satisfied.

$$
\phi(t) \leqq \psi(t)+c \int_{0}^{t} \tau^{l-1} \phi(\tau) d \tau
$$

where $c$ and $l$ are positive constants. Then we obtain

$$
\phi(t) \leqq \psi(t)+c \int_{0}^{t} \tau^{l-1} \psi(\tau) \exp \left\{\frac{c}{l}\left(l^{l}-\tau^{l}\right)\right\} d \tau
$$

Proof. Let $\Phi(t)$ be $\Phi(t)=\int_{0}^{t} \tau^{l-1} \phi(\tau) d \tau$, then

$$
\frac{d}{d t} \Phi(t)-c t^{l-1} \Phi(t) \leqq t^{l-1} \psi(t)
$$

Hence we can easily see (A.8).

Q.E.D.

Lemma $\mathbb{A}-5$. Let $i_{1}, \ldots, i_{n}(n=1,2, \ldots)$ be elements of $\{1,2, \ldots, m-1\}$. Then there exist constants $A_{1}, R_{1}$ such that

$$
n^{i_{1}+\cdots+i_{n}} \leqq A_{1} R_{1}^{n} 1^{i_{1}} 2^{i_{2} \cdots} n^{i_{n}} .
$$

Proof. Put $S=n^{i_{1}+\cdots+i_{n}} / 1^{i_{1}} 2^{i_{2} \cdots} n^{i_{n}}$. Then

$$
\begin{aligned}
S & =\left(\frac{n}{1}\right)^{i_{1}}\left(\frac{n}{2}\right)^{i_{2}} \times \cdots \times\left(\frac{n}{n}\right)^{i_{n}} \\
& \leqq\left(\frac{n}{1}\right)^{m-1}\left(\frac{n}{2}\right)^{m-1} \times \cdots \times\left(\frac{n}{n}\right)^{m-1}=\left(\frac{n^{n}}{n !}\right)^{m-1} .
\end{aligned}
$$

Stirling's formula yields 


$$
n ! \sim \sqrt{2} n^{n+1 / 2} e^{-n} \text { as } n \rightarrow \infty .
$$

Therefore there exist some constants $A_{1}, R_{1}$ such that $S \leqq A_{1} R_{1}^{n}$.

Q. E. D.

Lemma A-6. Let $i_{1}, \ldots, i_{n} \in\{1,2, \ldots, m-1\}$ for $n=1,2, \ldots$ and $0 \leqq q \leqq 1$. Then the following inequality holds.

$$
\left\{q\left(i_{1}+\cdots+i_{n}\right)\right\} ! \leqq A_{2} R_{2}^{n} n^{q\left(i_{1}+\cdots+i_{n}\right)}
$$

for some constants $A_{2}, R_{2}>0$ which are independent of $n$.

Proof. There exists $\hat{A}_{2}>0$ so as $x ! \leqq \hat{A}_{2} x^{x}$ for any $x>0$. Therefore

$$
\begin{aligned}
\left\{q\left(i_{1}+\cdots+i_{n}\right)\right\} ! & \leqq \hat{A}_{2}\left\{q\left(i_{1}+\cdots+i_{n}\right)\right\}^{\left(i_{1}+\cdots+i_{n}\right) q} \\
& \leqq \hat{A}_{2}\{q(m-1) n\}^{\left(i_{1}+\cdots+i_{n}\right) q} \\
& \leqq \hat{A}_{2}\{q(m-1)\}^{(m-1) n q} n^{\left(i_{1}+\cdots+i_{n}\right) q}
\end{aligned}
$$

Let $A_{2}=\hat{A}_{2}, R_{2}=\{q(m-1)\}^{(m-1) q}$, then we have (A-10).

Q.E.D.

Lemma A-7. Let $i_{1}, \ldots, i_{n}(n=1,2, \ldots)$ be elements of $\{1,2, \ldots, m-1\}$ and $0 \leqq q \leqq 1$. Then there exists $A_{3}, R_{3}, R^{\prime}$ which are independent of $r \geqq 0$ such that

$$
w_{r+\left(i_{1}+\cdots+i_{n}\right)}(s, t, R) \leqq A_{3} R_{3}^{n} n^{\left(i_{1}+\cdots+i_{n}\right) q \kappa} w_{r}\left(s, t, R^{\prime}\right)
$$

Proof. From the definition in $\S 4$,

$$
\begin{aligned}
w_{r+\left(i_{1}+\cdots+i_{n}\right) q}(s, t, R)= & R^{r+\left(i_{1}+\cdots+i_{n}\right) q}\left\{r+q\left(i_{1}+\cdots+i_{n}\right)\right\} !^{k} \\
& \times t^{s} \exp \left(K\left\{r+\left(i_{1}+\cdots+i_{n}\right) q\right\}^{*} t^{l}\right) .
\end{aligned}
$$

Let us note the following facts.

$$
\left\{r+\left(i_{1}+\cdots+i_{n}\right) q\right\} ! \leqq 2^{r+q\left(i_{1}+\cdots+i_{n}\right)}\left\{q\left(i_{1}+\cdots+i_{n}\right)\right\} ! r !
$$

$$
\left(i_{1}+\cdots+i_{n}\right) q \leqq(m-1) n
$$

$$
\left\{r+\left(i_{1}+\cdots+i_{n}\right) q\right\}^{*} \leqq r^{*}+(m-1) n
$$

Then it follows from Lemma A-4 that

$$
\left\{q\left(i_{1}+\cdots+i_{n}\right)\right\} ! \leqq A_{2} R_{2}^{n} n^{\left(i_{1}+\cdots+i_{n}\right) q} .
$$

Therefore we obtain

$$
\begin{aligned}
w_{r+\left(i_{1}+\cdots+i_{n}\right)}(s, t, R) \leqq & A_{2}^{\kappa}\left\{2^{(m-1) \kappa} R_{2}^{\kappa} R^{(m-1)} e^{K(m-1) t^{l}}\right\}^{n} \\
& \times n^{\left(i_{1}+\cdots+i_{n}\right) q \kappa} w_{r}\left(s, t, 2^{\kappa} R\right) .
\end{aligned}
$$

Q.E.D. 


\section{References}

[1] R. Beals, Hyperbolic equations and systems with multiple characteristics, Arch. Rat. Mech. Anal., 48 (1972), 123-152.

[2] M. S. Baouendi and C. Goulaouic, Cauchy problems with characteristic initial hypersurface, Comm. Pure Appl. Math., 26 (1973), 455-475.

[3] M. D. Bronshtein, The parametrix of the Cauchy problem for hyperbolic operators with characteristics of variable multiplicity, Funk. Anal. Ego Pril., 10 (1976), 83-84.

[4] K. Igari, An admissible data class of the Cauchy problem for non-strictly hyperbolic operators, J. Math. Kyoto Univ., 21 (1981), 351-373.

[5] V. Ja. Ivrii, Correctness of the Cauchy problem in Gevrey classes for nonstrictly hyperbolic operators, Math. Sb., 96 (1975), 390-413. (Russian; English translation in Math. USSR Sb.)

[6] - Well-posedness in projective Gevrey classes of the Cauchy problem for nonstrictly hyperbolic operators, Sovict Math. Dokl., 16 (1975), 570-573.

[7] K. Kajitani, Cauchy problem for nonstrictly hyperbolic systems in Gevrey classes, to uppear.

[8] H. Komatsu, Linear hyperbolic equations with Gevrey coefficients, J. Math. Pures Appl. 59 (1980), 145-185.

[9] J. Leray and Y. Ohya, Équations et systèmes Non-linéaires, hyperboliques non-stricts, Math. Annalen, 170 (1967), 167-205.

[10] Y. Ohya, Le problème de Cauchy pour les équations hyperboliques à caractéristique multiple, J. Math. Soc. Japan, 16 (1964), 268-286.

[11] O. Oleinik, On the Cauchy problem for weakly hyperbolic equations, Comm. Pure Appl. Math., 23 (1970), 569-586.

[12] S. Steinberg, Existence and uniqueness of the solutions of hyperbolic equations which are not necessarily strictly hyperbolic, J. Diff. Eq., 17 (1975), 119-153.

[13] H. Tahara, Singular hyperbolic systems, III. On the Cauchy problem for Fuchsian hyperbolic equations, J. Fac. Sci. Univ. Tokyo Set. IA, 27 (1980), 466-507.

[14] J. M. Trepreau, Le probleme de Cauchy hyperbolique dans les classes d'ultrafonctions et d'ultradistributions, Comm. in P.D.E., 4 (1979), 339-387.

[15] H. Uryu, The Cauchy problem for weakly hyperbolic equations, Comm. in P.D.E., 5 (1980), 23-40.

[16] Uniqueness for the characteristic Cauchy problem and its applications, Tokyo J. Math., 5 (1982), 117-136.

[17] S. Wakabayashi, The Cauchy problem for operators with constant coefficient hyperbolic principal part and propagation of singularities, Japanese J. Math., 6 (1980), 179-228. 
